WORKING PAPER - NO. 2020-57

\title{
Controlling Epidemic Spread: Reducing Economic Losses with Targeted Closures
}

John R. Birge, Ozan Candogan, and Yiding Feng

MAY 2020

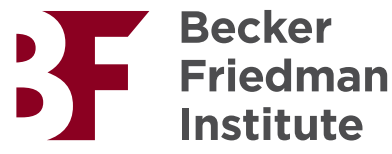




\title{
Controlling Epidemic Spread: Reducing Economic Losses with Targeted Closures
}

\author{
John R. Birge*, Ozan Candogan*, and Yiding Feng ${ }^{\dagger}$ \\ *Booth School of Business, University of Chicago. \\ ${ }^{\dagger}$ Department of Computer Science, Northwestern University. \\ \{john. birge@chicagobooth. edu, ozan. candogan@chicagobooth. edu, \\ yidingfeng2021@u.northwestern.edu\}
}

May 18, 2020

(Preliminary)

\begin{abstract}
We propose a spatial epidemic spread model to study the Covid-19 epidemic. In our model, a city consists of multiple neighborhoods, each of which has five disease compartments (susceptible/exposed/infected clinical/infected subclinical/recovered). Due to the movement of individuals across neighborhoods (e.g., commuting to work), the infections in one neighborhood can trigger infections in others. We consider the problem of a planner who reduces the economic activity in a targeted way to curb the spread of the epidemic. We focus both on the regime with a small number of infections and the regime with a large number of infections, and provide a framework for obtaining the policies that induce the lowest economic costs.

We use the available data on individuals' movements, level of economic activity in different neighborhoods, and the state of the epidemic to apply our framework to the control of the epidemic in NYC. Our results indicate that targeted closures can achieve the same policy goals at substantially lower economic losses than city-wide closure policies. In addition, to curb the spread of the epidemic in NYC, coordination with other counties is paramount. Finally, the optimal policy (under different scenarios) promotes some level of economic activity in Midtown Manhattan locations (due to their economic importance) while imposing closures in many other neighborhoods in the city (to curb the spread of the disease). Contrary to what might be intuitively expected, and due to the spatial aspect of the epidemic spread, neighborhoods with higher level of infections should not necessarily be the ones exposed to the most stringent economic closure measures.
\end{abstract}




\section{Introduction}

The Covid-19 pandemic has a global impact, with almost 4M confirmed cases and 278K deaths as of May 11, 2020 (BNO, 2020). It has also created unprecedented economic damage, with the global economy projected to contract by $3 \%$ (IMFBlog, 2020). To slowdown the spread of the disease many countries have imposed travel restrictions, which impact more than $90 \%$ of the global population (Pew Research, 2020). In addition, states, cities, and local governments have undertaken various non-pharmaceutical interventions (NPI), ranging from requiring residents to practice social distancing to lockdowns, and from ordering the residents to shelter-in-place to closing schools, non-essential services and businesses, and public spaces (Keystone, 2020). These interventions have also led to a substantial economic loss, bringing the unemployment numbers in the US to 23.1M individuals in April 2020 (U.S. Bureau of Labor Statistics, 2020).

Is it possible to curb the spread of the disease while minimizing the induced economic losses? In this paper, we investigate the answer to this question. So far many cities have instituted city-wide suspension of economic activity to varying degrees. However, the spread of the disease relies on human-to-human contact and has an inherent spatial nature, in which infected individuals potentially infect others in locations/neighborhoods they have visited. Thus, it may be possible to target the "hubs" in a city and institute the closure of public spaces and businesses in such locations so as to reduce the spread of the disease, while resuming economic activity elsewhere in the city. This could simultaneously reduce the economic losses and curb the spread of the disease. What is the optimal way to implement such targeted interventions?

To address this question, we propose a spatial epidemic spread model, which explicitly accounts for the spillovers of infections across different neighborhoods in a city. We focus on two regimes that capture different disease prevalence scenarios. In the first regime, the number of infected individuals is possibly large. The social planner seeks an economic closure policy that ensures that the infections in all neighborhoods decrease. Her optimal policy is the one that fulfills the aforementioned policy goal at the lowest possible economic cost. Next, we focus on the regime where the number of infected individuals is small. This regime is relevant when deciding how to shut down the economy after the emergence of a new infectious disease, as well as how to reopen the economy after an existing disease is nearly eradicated. The planner again controls the level of economic activity of each neighborhood, subject to the requirement that the permitted level of economic activity does not trigger an epidemic. Once again the objective of the planner is to find a policy that satisfies this requirement at the lowest possible economic cost. We provide convex optimization formulations of the planner's problem in both cases. We then focus on New York City, and use data on the movements of individuals within the city and disease-specific parameters for Covid-19 to model the spatial evolution of the epidemic at the zip code level. Finally, we use our optimization framework to obtain optimal intervention of the planner in both of the regimes described above. We shed light on the optimal targeting policies of the planner and discuss which neighborhoods should experience slowdown in economic activity.

Our epidemic spread model builds on the metapopulation/patch models from the mathematical epidemiology literature (Allen et al., 2008; Martcheva, 2015; Brauer et al., 2019), and captures the spatial nature of epidemic spread explicitly. In particular, in our model a 
city is divided into $n$ neighborhoods. Each neighborhood has a fixed population of individuals who reside there. Each of these subpopulations are subdivided into five compartments. A member of the subpopulation can be: (i) susceptible, (ii) exposed (infected but not infectious yet), infected (iii) with or (iv) without clinical symptoms, (v) recovered. Susceptible individuals in a given neighborhood can become infected through contact with an infected agent (that belongs to compartment (iii) or (iv)). The disease dynamics among these compartments are similar to the classic SEIR model (see, e.g., Brauer et al. (2019)), with the main difference being that the infected agents do not always show clinical symptoms. We allow the subclinical infectious populations to have a different infection rate than the clinical ones.

The individuals who reside in a neighborhood may spend some of their time in another neighborhood, e.g., due to work or social activities. Susceptible individuals from a neighborhood "mix" with other individuals in any of the neighborhoods in which they spend time, and they can get infected there. We model the aggregate amount of time that the members of each subpopulation spend in different neighborhoods, and characterize the amount of "mixing" between infected and susceptible agents that takes place in these neighborhoods. We then use this to characterize the rate with which the susceptible agents from a given neighborhood get infected. The model allows the susceptible agent from neighborhood $i$ to get infected by an infected agent from neighborhood $j$ when they are both spending time in neighborhood $k$ (where $i, j, k$ can be identical or different). Thus, the amount of time the members of each subpopulation spend in different neighborhoods plays a key role in the evolution of the epidemic.

We represent the aforementioned "flow" of subpopulations across different neighborhoods with a directed weighted network. This network, the populations of different neighborhoods, the initial disease state (if a nonzero number of individuals are infected), epidemic parameters governing the rates of infection and recovery, as well as the baseline economic activity in each neighborhood are the primitives of our model. Given these primitives, we focus on the decision problem of a social planner who can reduce the economic activity in each neighborhood. Formally, the planner chooses a scalar $x_{i} \in[0,1]$ that captures the permitted level of economic activity within each neighborhood $i$ relative to the baseline level. We assume that a fraction $\left(1-x_{i}\right)$ of the economic activity in neighborhood $i$ is suspended, thereby causing a proportional economic loss in this neighborhood. In this case, we assume that a fraction $\left(1-x_{i}\right)$ of all of the agents who would otherwise spend time in this neighborhood and mix with others instead stay at home, thereby not infecting others or getting infected. Note that because of the spatial structure of the epidemic spread discussed above, the slowdown of economic activity in one neighborhood impacts the infections among individuals who reside in this neighborhood, as well as the individuals who reside elsewhere but spend time in this neighborhood.

The objective of the planner is to minimize the total economic loss across all neighborhoods, while guaranteeing either that, as a result of the choice of $\left\{x_{i}\right\}$, the number of infected agents decreases in all neighborhoods with infection individuals (in the first regime), or that the number of infected cannot increase drastically (in the second regime). The second requirement is especially relevant in the regime where only a small number of agents are infected, and is formally expressed as the asymptotic stability of the no-disease equilibrium 
(which is equivalent to having the effective reproduction rate $\mathscr{R}_{e}$ satisfying $\mathscr{R}_{e}<1$ ). ${ }^{1}$

We show that the problem of the planner is tractable in both regimes. In particular, in the first regime the problem can be formulated as a linear program, whereas in the second one it can be formulated as a semidefinite program. Because of the spatial nature of the epidemic, in both regimes, the optimal levels of economic activity in different neighborhoods are interdependent. We shed light on which neighborhoods should be targeted with closures and how this depends on the underlying network structure.

We illustrate our approach by using data from NYC. We define neighborhoods in terms of the ZIP Code Tabulation Areas (ZCTA) in NYC. In addition, we include neighboring counties that have a notable flow of individuals to/from NYC. We rely on data from SafeGraph to capture the movement of individuals across neighborhoods (see SafeGraph, 2020) $!^{2}$ This allows us to construct the underlying network. Combining this network with the spread parameters for Covid-19 presented in the literature, we complete our spatial epidemic spread model. Leveraging the ZIP Codes Business Patterns data from the Census Bureau we capture the number of individuals employed in each ZCTA, and use this as our measure of the baseline economic value of the relevant ZCTA. Under this assumption the planner's problem can equivalently be interpreted as minimizing the total (temporary) unemployment induced by the slowdown of economic activity, while ensuring that the spread of the disease is curbed. We assume that the planner can influence the economic activity in NYC and not the neighboring counties. The economic activity in the neighboring counties is exogenously fixed, and we study how the optimal solution changes as a function of this activity level.

We first focus on the ZCTA level infection data from NYC on April 18 (see NYC Health, 2020), when the total number of reported cases in the city is relatively large (with a cumulative number of 128,777 since the disease was first seen in the city, with 72,388 of the cases being from the preceding two weeks). This corresponds to our first regime, and we investigate the optimal targeted economic restrictions. We observe that it is optimal for the planner to permit a limited level of economic activity in a number of ZCTAs in the midtown area, while suspending it in many of the other neighborhoods. At a high level, this structure of the policy is driven by the relatively high economic value (in terms of employment numbers) of these ZCTAs. At the same time, due to the spatial nature of the epidemic spread, resuming the economic activity in this area contributes to infections in different neighborhoods. Hence, due to the constraint that the infections should decrease in all neighborhoods, the planner suspends economic activity in the majority of the remaining ZCTAs.

In this problem, the economic loss in NYC induced by the optimal policy quite heavily depends on the economic activity level in the neighboring counties. For instance, if the neighboring counties completely suspend economic activity, NYC can achieve the policy goal of reducing infections in all neighborhoods by resuming $48.8 \%$ of the overall economic activity. By contrast, if the neighboring regions (i.e. counties outside NYC) resume $80 \%$ of their economic activity, the permissible economic activity level in NYC becomes $40.0 \%$.

\footnotetext{
${ }^{1}$ The question in the second regime is similar to the one posed in Budish (2020), where the focus is on understanding what activities maximize social welfare while satisfying the $\mathscr{R}<1$ constraint. In our setting we model the spatial nature of epidemic spread and focus on a similar constraint and objective to explore the benefit of targeted economic closure policies.

${ }^{2} \mathrm{SafeGraph}$ is a data company that aggregates anonymized location data from numerous applications in order to provide insights about physical places.
} 
This observation highlights the need for coordination between governing bodies (of different counties and states) in the control of the Covid-19 epidemic.

An important question is how much the planner gains from targeted closures, as opposed to uniform city-wide closures that impose the same level of economic restrictions in all neighborhoods. The latter class of policies are of interest as they may be perceived as being more fair and possibly easier to implement. We show that imposing uniform closures decreases the amount of allowable economic activity by a factor of 4.36 (or more) under the original policy goal (of achieving a decrease in the infections in all neighborhoods). This observation highlights the importance of considering targeted closures to curb the spread of the epidemic while limiting the induced economic losses.

We then turn our attention to the small infection regime. In this regime, we see that to prevent a larger epidemic and to limit the associated economic cost, the planner finds it optimal to resume economic activity (almost fully) in some neighborhood (e.g., Lower Manhattan, Midtown Manhattan), while allowing for partial levels of economic activity elsewhere in the city. Relative to the large epidemic regime, as expected, the level of permissible economic activity (that guarantees the policy goal) is substantially higher (93.0\% vs $48.8 \%$ when there is no economic activity in the neighboring counties). Furthermore, the optimal policy for NYC, as well as its economic cost, is relatively insensitive to the level of economic activity in neighboring regions for as long as their economic activity levels are not too high. On the other hand, if the neighboring regions have substantial economic activity, it is not possible to adjust the economic activity in NYC in a way that guarantees that an epidemic can be prevented. In this case, the infections in the neighboring counties and their spillovers are sufficient to infect many individuals in NYC and start an epidemic.

Motivated again by fairness considerations, we explore the uniform policies that require economic activities to continue at the same rate in all regions in the small infection regime. We observe that the uniformity requirement once again induces additional economic losses. In order to achieve the same policy goal, permissible economic activity drops from targeted policies to uniform policies $(93.0 \%$ vs $81.5 \%$ when there is no economic activity in the neighboring counties). This finding once again highlights the value of targeted closures in the small infection regime.

Even though we focus on NYC to illustrate our framework, it is worth noting that our approach is also relevant at a more macro level. For instance, the spatial effects can be relevant for epidemic spread between states or countries (e.g., due to trips individuals take across states). Taking the associated disease spillovers into account, a country can target different states with different economic closure measures and minimize the economic cost of achieving certain policy goals. The approach presented in this paper is readily applicable to such settings.

Our analysis opens up a number of future directions related to the modeling of spatial epidemics and their control with appropriate targeting measures. We present our model and optimization framework in Section 2. We then discuss the application of our framework to NYC in Section 3, where we also discuss various data sources that are used to construct the primitives of our model. We conclude in Section 4 and we detail the aforementioned future directions related to this work there. 


\section{Related Literature}

Spatial aspects of epidemic spread play an important role in prediction, estimation, and understanding of disease transmission (Anderson et al., 1992; McNeill and McNeill, 1998; Allen et al., 2008; Martcheva, 2015; Brauer et al., 2019). Different models from epidemiology are proposed to analyze the spatial spread of diseases and their control (Hethcote, 1976 ; Hethcote and Van Ark, 1987; Diekmann et al., 1990; Van den Driessche and Watmough, 2002). Spatial effects have received attention in the recent literature that studies Covid-19 as well. Using data on population flows (from Wuhan, China) Jia et al. (2020) propose a spatiotemporal "risk source" model to forecast confirmed cases and identify high-transmission-risk locales. This model sheds light on the spread of Covid-19 and its growth pattern in China. Similarly, Chinazzi et al. (2020) use a metapopulation model and project the impact of travel limitations on the national and the international spread of the epidemic.

The spatial nature of epidemic spread admits a natural network representation, where nodes correspond to different locations and edges encode travel of individuals between these locations. Other network models that focus on an underlying social network and contact between the individuals in the social network have also proven useful in the study of epidemics. Jackson and López-Pintado (2013) study how social network structure impacts diffusion, with a focus on the effect of homophily. Ogura and Preciado (2016, 2017) and Ogura et al. (2019) introduce an epidemic model over adaptive state-dependent networks, analyze the epidemic threshold for the disease-free equilibrium, and propose optimal algorithmic tuning on the adaptation rates to achieve the disease-free equilibrium. Drakopoulos and Zheng (2017) study the problem of identifying network effects in contagion processes, and introduce an instrumental variable approach, based on a spatiotemporally lagged version of the observed data. Drakopoulos et al. (2014, 2017) consider the problem of allocating limited (curing) resources to minimize the expected time of the epidemic extinction in an SIS epidemic model with a given network structure. Nowzari et al. (2015) propose a generalized epidemic model over networks and study the optimal policy that guarantees global exponential stability of the disease-free equilibrium while minimizing the total induced cost. In most of models discussed here, nodes correspond to individuals and the disease can be transmitted from one individual to another. By contrast, in the spatial epidemic model we consider, each node (location) has a population of susceptible and infected individuals. The infected individuals from one node can travel to another node, and meet there with susceptible individuals from a third node and infect them. This structure leads to a richer set of networked interactions.

In recent literature, various estimation, testing, and control questions related to Covid19 have also been studied. Kaplan (2020a) presents a probabilistic model for estimating the effectiveness of isolation and quarantine within a community and applies it to data from Wuhan. Drakopoulos and Randhawa (2020) consider the allocation of limit testing to strategic agents, and study the optimal policy for the social planner. Kaplan (2020b) presents decision-making models and solutions implemented in Yale University, the Yale New Haven Hospital, and the State of Connecticut during the early phase of the Covid-19

outbreak. Alvarez et al. (2020) study the optimal lockdown policy for minimizing the loss of long-term social welfare due to fatalities in a single population. Acemoglu et al. (2020) generalize this problem from a single-population model to a heterogeneous SIR model where different subpopulations (e.g., age groups) are susceptible to different risks (i.e., infection, 
hospitalization, and fatality rates vary between subpopulations). Glover et al. (2020) consider a similar problem where the population is partitioned into age and sector groups, and a social planner chooses lockdown policies and how to redistribute income across the groups to optimize long-term social welfare. Lipton and Lopez de Prado (2020) and Gershon et al. (2020) also focus on heterogeneous SEIR models with populations with different risk levels, and analyze how to manage the progression of the disease without exceeding the healthcare capacity or creating undue economic burden. Utilizing cross-county data on face mask use, Abaluck et al. (2020) argue that the universal adoption of cloth face masks by the public slows the disease growth rate of cases and deaths. Furthermore, the use of cloth face masks by the public does not create a shortage of medical masks by healthcare workers. The authors shed light on the economic value generated by using masks.

Covid-19 has already impacted the economic activity around the globe immensely. Various economic impacts of the disease (e.g., on financial markets, fiscal policies, employment and wage, etc.) have been explored in the recent literature (Mulligan, 2020, Coibion et al. 2020; Gormsen and Koijen, 2020; Guerrieri et al., 2020; Baker et al., 2020a, b c; He and Liu, 2020; Hanson et al., 2020). Budish et al. (2020) discuss an economic policy response to the Covid-19 crisis, and offer frameworks and guiding principles to deal with the crisis. Budish (2020) proposes focusing on minimizing the social welfare loss while taking the appropriate steps to ensure that the disease is contained. The present paper adopts a similar approach, and proposes targeted closures as a lever for achieving this goal.

\section{$2 \quad$ Model and Preliminary Results}

\subsection{A Spatial Compartmental Model of Epidemics}

We consider a city with a set $\mathcal{N}=[n]:=\{1, \ldots, n\}$ of neighborhoods. We denote the population of neighborhood $i \in[n]$ by $N_{i}$. We assume that members of neighborhood $i$ spend a fraction $\tau_{i j}$ of their time in neighborhood $j$ (in aggregate). Here $\sum_{j} \tau_{i j} \leq 1$, and we let $1-\sum_{j} \tau_{i j}$ capture the fraction of time during which the members of population $i$ are isolated (and hence cannot infect others or be infected by them). As we will formalize shortly, the amount of time the members of a population spend in other neighborhoods impacts the contact between susceptible/infected agents and subsequently the rate of spread of the epidemic. We represent this setting using a directed weighted network, where the nodes correspond to the neighborhoods and $\left\{\tau_{i j}\right\}$ correspond to the edge weights. In what follows, we alternatively refer to the neighborhoods as nodes.

We assume that each member of the population in location $i$ belongs to one of the five compartments with populations $S_{i}, E_{i}, I_{i}^{c}, I_{i}^{s c}, R_{i}$ that satisfy $S_{i}+E_{i}+I_{i}^{c}+I_{i}^{s c}+R_{i}=N_{i}$. Here $S_{i}$ denotes the population of susceptible individuals at $i$ (who can get infected if they come in contact with an infected agent), $E_{i}$ denotes the population of agents that are exposed to the disease but who are not infectious yet (e.g., since the infection is in the incubation period). These agents subsequently move to one of the two infected compartments. The first of these compartments, with population $I_{i}^{c}$, is referred to as the infected clinical population and captures the individuals who are infected and show symptoms. The second compartment has population $I_{i}^{s c}$ and is referred to as the infected subclinical population. The agents in 
both compartments are infectious but possibly with different infection rates. The size of the latter compartment may not be readily available to a social planner, which may impact the disease mitigation strategies. The final compartment, with population $R_{i}$, captures the infected agents that recover from the disease and become immune to it. They have no impact on the future progression of the disease. Note that in this formulation we do not model the disease-related deaths as they are a small fraction of the total population. Such deaths could alternatively be included in the $R_{i}$ population, as they have no impact on the future evolution of the disease and in that sense are removed from the system.

The evolution of the disease is governed by six parameters, which are listed next:

- $\beta$, the effective contact rate, captures the rate with which an infected agent infects others. It can be viewed as the product of the contact rate (the number of contacts an individual has with others) times the transmission risk (the probability with which a meeting between an infected individual and a susceptible individual results in an infection).

- $\kappa$, the infectious rate, captures how quickly the exposed individuals move to the infected compartment.

- $\rho \leq 1$, the clinical rate, captures the fraction of infected agents who show clinical symptoms (and join the $I_{i}^{c}$ population after leaving the $E_{i}$ population).

- $\alpha \leq 1$, the discount factor, captures the reduced risk of infections in meetings between susceptible and subclinical (as opposed to clinical) agents.

- $\gamma$, the removal rate, captures the rate with which the agents in the infected compartments move to the recovered compartment.

- $\mu$ is the natural birth/death rate in the population. For our analysis, we will assume that $\mu \approx 0$.

Using these parameters and the spatial structure of the epidemic outlined earlier, for all $i \in[n]$ the system of differential equations that govern the evolution of the population of different compartments can now be stated as follows:

$$
\begin{aligned}
& S_{i}^{\prime}=\mu N_{i}-\beta \sum_{j} \underbrace{S_{i} \tau_{i j}}_{\text {Time agent } \in S_{i} \text { spends at } j}(\underbrace{\frac{\sum_{k} I_{k}^{c} \tau_{k j}}{\sum_{k} N_{k} \tau_{k j}}}_{\text {Fraction of clinical infected at } j}+\alpha \underbrace{\frac{\sum_{k} I_{k}^{s c} \tau_{k j}}{\sum_{k} N_{k} \tau_{k j}}}_{\text {subclinical infected at } j})-\mu S_{i} \\
& E_{i}^{\prime}=\beta \sum_{j} \underbrace{S_{i} \tau_{i j}}_{\text {Time agent } \in S_{i} \text { spends at } j}(\underbrace{\frac{\sum_{k} I_{k}^{c} \tau_{k j}}{\sum_{k} N_{k} \tau_{k j}}}_{\text {Fraction of clinical infected at } j}+\alpha \underbrace{\frac{\sum_{k} I_{k}^{s c} \tau_{k j}}{\sum_{k} N_{k} \tau_{k j}}}_{\text {subclinical infected at } j})-(\mu+\kappa) E_{i} \\
& I_{i}^{c \prime}=\rho \kappa E_{i}-(\mu+\gamma) I_{i}^{c} \\
& I_{i}^{s c l}=(1-\rho) \kappa E_{i}-(\mu+\gamma) I_{i}^{s c} \\
& R_{i}^{\prime}=\gamma I_{i}^{c}+\gamma I_{i}^{s c}-\mu R_{i} .
\end{aligned}
$$


A few comments are in order. First, observe that susceptible agents from neighborhood $i$ can get infected at neighborhood $i$ as well as in other neighborhoods. For instance, they spend a fraction $\tau_{i j}$ of their time in neighborhood $j$ (in aggregate), and they get infected at a rate proportional to this quantity. Second, as is common in classic epidemic models, the rate of infection is also proportional to the fraction of infected agents that are mixing in a neighborhood. On the other hand, this term now also depends on the time agents spend in different neighborhoods. This dependence is often found in spatial epidemic models, and is similar to Post et al. (1983). To see the dependence clearly, consider neighborhood $j$, and observe that, after taking into account the fact that agents from different neighborhoods visit $j$, we conclude that this neighborhood has an effective mass of $\sum_{k} N_{k} \tau_{k j}$ individuals that are mixing at $j$. Of these, $\sum_{k} I_{k}^{c} \tau_{k j}$ are infected clinical and $\sum_{k} I_{k}^{s c} \tau_{k j}$ are infected subclinical agents. The ratio of infected agents (of both types) to the total effective population mixing at $j$ determines the rate with which the susceptible agents (from different neighborhoods) are infected in this neighborhood. Third, the last term clarifies the role of the discount factor $\alpha$ : a contact with a subclinical agent is less likely to result in an infection. Fourth, all susceptible agents that get the disease initially move to the exposed compartment, but only a fraction $\rho$ of these develop clinical symptoms (and join $I_{i}^{c}$ ), while the remaining become infectious without any clinical symptoms. The rate with which exposed agents move to one of the infected compartments is $\kappa$. Fifth, all infected agents move to the recovered compartment at a rate of $\gamma$. Finally, the new births occur at a rate of $\mu N_{i}$ (and the newborns join the susceptible pool) while in each compartment natural deaths reduce the population at a rate of $\mu$. As a result of this structure, we obtain $S_{i}^{\prime}+E_{i}^{\prime}+I_{i}^{c^{\prime}}+I_{i}^{s c^{\prime}}+R_{i}^{\prime}=0$ and $N_{i}=S_{i}+E_{i}+I_{i}^{c}+I_{i}^{s c}+R_{i}$.

For our analysis of the large infection regime (Section 2.2), the natural deaths/births can be completely ignored (and $\mu$ can be set equal to zero). In the analysis of the small infection regime, we will explore the asymptotic stability of the disease-free equilibrium and for well posedness of this question, we will assume that $\mu>0$ (but still $\mu \approx 0$ ). It is also worthwhile to point out that, for the large infection regime, the assumption that the total population remains constant does not play any role and the disease-related deaths can be incorporated into the model in a straightforward way.

We assume that a social planner can restrict the economic activity in each neighborhood in order to control the spread of the epidemic. Let $x_{i} \in[0,1]$ be the level of permitted economic activity in neighborhood $i$. When $x_{i}<1$, this implies that a fraction $1-x_{i}$ of the economic activity in neighborhood $i$ as being suspended (e.g., by closing a fraction of businesses and/or public places). In this case, we assume that agents from $j$ spend $1-x_{i}$ times less time in $i$; i.e., $\tau_{j i}$ in the model is replaced with $\tau_{j i} x_{i}$. Note that given $\left\{x_{i}\right\}$, the 
dynamics become:

$$
\begin{aligned}
S_{i}^{\prime} & =\mu N_{i}-\beta \sum_{j} S_{i} \tau_{i j} x_{j} \frac{\sum_{k}\left(I_{k}^{c}+\alpha I_{k}^{s c}\right) \tau_{k j}}{\sum_{k} N_{k} \tau_{k j}}-\mu S_{i} \\
E_{i}^{\prime} & =\beta \sum_{j} S_{i} \tau_{i j} x_{j} \frac{\sum_{k}\left(I_{k}^{c}+\alpha I_{k}^{s c}\right) \tau_{k j}}{\sum_{k} N_{k} \tau_{k j}}-(\mu+\kappa) E_{i} \\
I_{i}^{c \prime} & =\rho \kappa E_{i}-(\mu+\gamma) I_{i}^{c} \\
I_{i}^{s c \prime} & =(1-\rho) \kappa E_{i}-(\mu+\gamma) I_{i}^{s c} \\
R_{i}^{\prime} & =\gamma\left(I_{i}^{c}+I_{i}^{s c}\right)-\mu R_{i} .
\end{aligned}
$$

If $x_{i}<1$, our model implies that a fraction $\left(1-x_{i}\right)$ of the individuals who would otherwise spend time in neighborhood $i$ instead stay at home and as a result they do not infect others (or become infected). This also applies to agents who reside in neighborhood $i$ : they also isolate themselves at home as opposed to mixing with others in neighborhood $i$. Note that due to the spatial nature of the epidemic spread, suspension of economic activity in neighborhood $i$ may reduce infection rates in other neighborhoods as well. For instance, the susceptible agents who reside in neighborhood $j$ but commute to neighborhood $i$ for work may cease to mix with other agents at $i$, which in turn reduces the infection rates in $j$.

We assume that suspending economic activity is economically costly. In particular, setting the economic activity level in location $i$ to $x_{i}$ induces an economic loss of $c_{i}\left(1-x_{i}\right)$, where $c_{i}$ is a fixed location-specific constant. We investigate the least costly ways of reducing the economic activity while ensuring that the spread of the epidemic is curbed.

\subsection{Large Infection Regime}

When the number of infected individuals is large, a natural policy goal for the planner is to choose permitted levels of economic activity so that the infections in all neighborhoods (weakly) decrease. Mathematically, we express this condition as $E_{i}^{\prime}+I_{i}^{c \prime}+I_{i}^{s c l} \leq 0$ for all $i$. Note that here we not only focus on the clinical and subclinical populations, but also on the exposed population. The motivation for this is that these agents become infectious with some delay; hence, in order to curb the spread of the disease, it is necessary to limit the number of such agents as well.

The problem of reducing infections in all neighborhoods, while incurring the lowest economic cost, can now be expressed as a simple linear program:

$$
\begin{aligned}
\max _{x_{i} \in[0,1]} & \sum_{i} c_{i} x_{i} \\
\text { s.t. } & \sum_{j} S_{i} \tau_{i j} x_{j} \frac{\sum_{k}\left(I_{k}^{c}+\alpha I_{k}^{s c}\right) \tau_{k j}}{\sum_{k} N_{k} \tau_{k j}} \leq \frac{\mu}{\beta} E_{i}+\frac{\mu+\gamma}{\beta}\left(I_{i}^{c}+I_{i}^{s c}\right) \quad \forall i .
\end{aligned}
$$

Here the constraint is obtained by summing (2)-(4) and imposing $E_{i}^{\prime}+I_{i}^{c \prime}+I_{i}^{s c \prime} \leq 0$ more explicitly. 
Remark 1. In our computational studies we set $\mu=0$. Choosing a small positive value has a negligible impact on the results. In addition, in (P1) we impose the constraint for $i$ such that $I_{i}^{c}+I_{i}^{s c}>0$. This is because, when $\mu=0$, the aforementioned constraint cannot hold unless there are infected individuals, i.e., the total number of exposed and infected individuals cannot decrease if there are no infected agents. It is worth pointing out that in the large infection regime this condition is likely to be satisfied for all neighborhoods. Finally, as mentioned earlier, the disease-related deaths can be incorporated into our framework. For instance, the compartment $\left\{R_{i}\right\}$ can be interpreted as the set of all recovered individuals and all individuals who die from the disease. A death rate can explicitly model the rate with which people recover vs. die from the disease. Our formulation can be readily adapted to this setting, and the cost of expected (future) deaths from the disease can also be included in our objective function.

Remark 2. Note that in the formulation presented in this section, we focus on a "snapshot" of infections at a point in time and impose the constraint that the infections decrease at that time. This requirement can also be used to define a heuristic dynamic policy for the control of the epidemic over time. For instance, given current infection numbers, it is possible to solve the relevant optimization problem and obtain the (current) optimal economic activity levels. After imposing this policy for a fixed amount of time, the problem can be resolved with the new populations of compartments that result from the policy. Similar steps can be repeated over time to adjust the policy as needed.

Remark 3. We focused on the policy goal of reducing infections in all neighborhoods. An alternative goal is to impose reduction in total infections citywide. An accompanying optimization formulation can easily be obtained by summing both sides of the constraints of (P1) over $i$. The associated optimal policy allows for substantial activity in Manhattan (due to its economic value), which in turn increases the infections there. The policy balances this by restricting activity elsewhere to achieve a net reduction in infections. However, since an increase in infections in Manhattan triggers future spread, such a policy could lead to difficulties in controlling the infection in the long run.

\subsection{Small Infection Regime: Preventing an Epidemic}

Another natural goal for the planner pertains to the setting where only a few agents are infected. In this regime, the planner may be interested in preemptively lowering economic activity so that the number of infected individuals does not drastically increase and trigger an epidemic. This regime is relevant when there is a new infectious disease impacting the population. It is also relevant when a large number of individuals are initially infected, but subsequently (e.g., due to quarantine measures) a majority of them recover, and the active infections dwindle. In this setting, ignoring the recovered agents (who have no impact on further infections), the evolution of the epidemic can be analyzed in a similar fashion to the setting where a new disease impacts the population. For simplicity of exposition, we will focus on the first setting. 
To study this regime formally, we first note that given $\left\{x_{i}\right\}$, an equilibrium of the dynamical system (1)-(5) is such that $I_{i}^{c}=I_{i}^{s c}=E_{i}=R_{i}=0$. We refer to this equilibrium as the disease-free equilibrium. We can view the case where only a few individuals are infectedd as a perturbation of the disease-free equilibrium. After such a perturbation, the system may evolve back to the disease-free equilibrium or deviate from it with a growing number of infections. Formally, the former alternative corresponds to the asymptotic stability of the disease-free equilibrium. In the second regime, we assume that the planner's policy goal is to choose $x=\left\{x_{i}\right\}$ in a way that ensures the asymptotic stability of the disease-free equilibrium. The optimal $\left\{x_{i}\right\}$ obtains this goal at the lowest possible economic cost.

For nonlinear systems, the asymptotic stability of an equilibrium can be characterized through the linearization of the dynamics around the equilibrium. More formally, for a dynamical system $\dot{y}=f(y)$ with equilibrium $y=0$, let $\partial f / \partial y$ denote the Jacobian of $f$ evaluated at $y=0$. If the real parts of the eigenvalues of the Jacobian are strictly less (greater) than one, then $y=0$ is asymptotically stable (unstable); see, e.g., Khalil (2002). Similarly, the asymptotic stability of the disease-free equilibrium for the dynamical system (1)-(5) can be studied using the associated Jacobian matrix. This is equivalent to using the next generation method (see, e.g., Allen et al., 2008; Martcheva, 2015) from the mathematical epidemiology literature, which adapts similar conditions to compartmental models of epidemic spread.

We proceed by deriving the aforementioned stability condition for our setting more explicitly. Define the following vectors:

$$
\mathscr{F}=\left(\begin{array}{c}
\beta S_{1} \sum_{j} \tau_{1 j} x_{j} \frac{\sum_{k}\left(I_{k}^{c}+\alpha I_{k}^{s c}\right) \tau_{k j}}{\sum_{k} N_{k} \tau_{k j}} \\
\vdots \\
\beta S_{n} \sum_{j} \tau_{n j} x_{j} \frac{\sum_{k}\left(I_{k}^{c}+\alpha I_{k}^{s c}\right) \tau_{k j}}{\sum_{k} N_{k} \tau_{k j}} \\
0 \\
\vdots \\
0 \\
0 \\
\vdots \\
0
\end{array}\right) \text { and } \mathscr{V}=\left(\begin{array}{c}
(\mu+\kappa) E_{1} \\
\vdots \\
(\mu+\kappa) E_{n} \\
-\rho \kappa E_{1}+(\mu+\gamma) I_{1}^{c} \\
\vdots \\
-\rho \kappa E_{n}+(\mu+\gamma) I_{n}^{c} \\
-(1-\rho) \kappa E_{1}+(\mu+\gamma) I_{1}^{s c} \\
\vdots \\
-(1-\rho) \kappa E_{n}+(\mu+\gamma) I_{n}^{s c}
\end{array}\right) .
$$

Observe that the right-hand sides of (2), (3), and (4) simply correspond to $\mathscr{F}-\mathscr{V}$. Viewing these vectors as functions of $\left(E_{1}, \ldots, E_{n}, I_{1}^{c}, \ldots, I_{n}^{c}, I_{1}^{s c}, \ldots, I_{n}^{s c}\right)$, the Jacobian of $\mathscr{F}$ and $\mathscr{V}$, evaluated in the disease-free equilibrium, can be expressed as follows:

$$
\begin{aligned}
F(x) & =\left(\begin{array}{ccc}
\mathbf{0} & \left(\beta N_{i} \sum_{k} \tau_{i k} x_{k} \frac{\tau_{j k}}{\sum_{l} N_{l} \tau_{l k}}\right)_{i \in[n], j \in[n]} & \left(\alpha \beta N_{i} \sum_{k} \tau_{i k} x_{k} \frac{\tau_{j k}}{\sum_{l} N_{l} \tau_{l k}}\right)_{i \in[n], j \in[n]} \\
\mathbf{0} & \mathbf{0} & \mathbf{0} \\
\mathbf{0} & \mathbf{0} & \mathbf{0}
\end{array}\right) \\
V & =\left(\begin{array}{ccc}
\operatorname{diag}_{n}(\mu+\kappa) & \mathbf{0} & \mathbf{0} \\
\operatorname{diag}_{n}(-\rho \kappa) & \operatorname{diag}_{n}(\mu+\gamma) & \mathbf{0} \\
\operatorname{diag}_{n}(-(1-\rho) \kappa) & \mathbf{0} & \operatorname{diag}_{n}(\mu+\gamma)
\end{array}\right)
\end{aligned}
$$


Here, $\mathbf{0}$ is an $n \times n$ matrix whose entries are equal to zero, and we use the shorthand notation $\operatorname{diag}_{n}(a)$ to denote an $n \times n$ diagonal matrix, whose diagonal entries are given by some scalar $a$.

Given a matrix $A$, let $\lambda_{1}(A)$ denote its largest eigenvalue (in absolute value). The asymptotic stability of the disease-free equilibrium is equivalent to

$$
\mathscr{R}_{e}:=\lambda_{1}\left(F(x) V^{-1}\right)<1 .
$$

The quantity on the left-hand side is often referred to as the effective reproduction rate of the disease, and the stability requirement puts this number at less than one. If the inequality is flipped, the disease-free equilibrium is unstable (see, e.g., Martcheva, 2015; Diekmann et al., 1990).

It can be shown that $V^{-1}$ is a matrix with nonnegative entries (see the proof of Lemma 2.1). In turn, this implies that $F(x) V^{-1}$ is a matrix with nonnegative entries. Thus, the PerronFrobenius theorem applies, and we conclude that the latter matrix has a real positive eigenvalue that is larger than all its eigenvalues in absolute value. Hence, $\lambda_{1}\left(F(x) V^{-1}\right)$ is real and the condition in $(6)$ is well posed.

In the small infection regime, the planner's policy goal can alternatively be stated as having $\mathscr{R}_{e}<1$. Note that in (6), the constraints imposed on $x=\left\{x_{i}\right\}$ are given in terms of the eigenvalues of a matrix. We proceed by providing an equivalent and more explicit statement of these conditions. In what follows, for a symmetric matrix $A$, we use the notation $A \succ 0$ to indicate that it is positive definite. We denote by $T$ the matrix whose $(i, j)$ th entry is $\tau_{i j}, \hat{N}$ the diagonal matrix with diagonal entries $\left\{N_{i}\right\}$, and by $\Lambda$ the diagonal matrix with diagonal entries $1 / \sum_{l} N_{l} \tau_{l k}$. All of these matrices are in $\mathbb{R}^{n \times n}$.

Lemma 2.1. Condition (6) is equivalent to $T X \Lambda T^{\prime} \prec \hat{N}^{-1} \frac{(\mu+\kappa)(\mu+\gamma)}{(\rho+\alpha(1-\rho)) \beta \kappa}$.

Proof. It can be seen by inspection that

$$
V^{-1}=\left(\begin{array}{ccc}
\operatorname{diag}_{n}\left(\frac{1}{\mu+\kappa}\right) & \mathbf{0} & \mathbf{0} \\
\operatorname{diag}_{n}\left(\frac{\rho \kappa}{(\mu+\kappa)(\mu+\gamma)}\right) & \operatorname{diag}_{n}\left(\frac{1}{\mu+\gamma}\right) & \mathbf{0} \\
\operatorname{diag}_{n}\left(\frac{(1-\rho) \kappa}{(\mu+\kappa)(\mu+\gamma)}\right) & \mathbf{0} & \operatorname{diag}_{n}\left(\frac{1}{\mu+\gamma}\right)
\end{array}\right) .
$$

Thus, it follows that $F(x) V^{-1}$ has the same non-zero eigenvalues as the following matrix:

$$
\frac{(\rho+\alpha(1-\rho)) \beta \kappa}{(\mu+\kappa)(\mu+\gamma)}\left(N_{i} \sum_{k} \tau_{i k} x_{k} \frac{\tau_{j k}}{\sum_{l} N_{l} \tau_{l k}}\right)_{i \in[n], j \in[n] .}
$$

Using these observations, we conclude that condition (6) is equivalent to $\lambda_{1}(\bar{F}(x))<$ $\frac{(\mu+\kappa)(\mu+\gamma)}{(\rho+\alpha(1-\rho)) \beta \kappa}$, where

$$
\bar{F}(x)=\left(\sum_{k} N_{i} \tau_{i k} x_{k} \frac{\tau_{j k}}{\sum_{l} N_{l} \tau_{l k}}\right)_{i j} .
$$

We rewrite this matrix as $\bar{F}(x)=\hat{N} T X \Lambda T^{\prime}$. Note that the symmetric matrix

$$
\hat{N}^{-1 / 2} \hat{N} T X \Lambda T^{\prime} \hat{N}^{1 / 2}=\hat{N}^{1 / 2} T X \Lambda T^{\prime} \hat{N}^{1 / 2}
$$


is obtained after a similarity transformation of $\hat{N} T X \Lambda T^{\prime}$; hence, the two matrices share the same eigenvalues. Thus, our condition is equivalent to $\lambda_{1}\left(\hat{N}^{1 / 2} T X \Lambda T^{\prime} \hat{N}^{1 / 2}\right)<\frac{(\mu+\kappa)(\mu+\gamma)}{(\rho+\alpha(1-\rho)) \beta \kappa}$, which can equivalently be written as

$$
T X \Lambda T^{\prime} \prec \hat{N}^{-1} \frac{(\mu+\kappa)(\mu+\gamma)}{(\rho+\alpha(1-\rho)) \beta \kappa} .
$$

Using this lemma, in the second regime, we formulate the planner's optimization problem as follows:

$$
\begin{aligned}
\max _{x_{i} \in[0,1]} & \sum_{i} c_{i} x_{i} \\
\text { s.t. } & T X \Lambda T^{\prime} \preceq \hat{N}^{-1} \frac{(\mu+\kappa)(\mu+\gamma)}{(\rho+\alpha(1-\rho)) \beta \kappa} .
\end{aligned}
$$

Note that in this formulation, as opposed to imposing positive definiteness, we impose positive semidefiniteness. This is equivalent to relaxing the planner's problem by taking a closure of the feasible region, and we do this to ensure the existence of an optimal solution to this problem. It can be trivially checked that given an optimal solution to this problem, multiplying $x_{i}$ by $(1-\epsilon)$ for all $i$ and $\epsilon>0$ is a feasible solution that guarantees that the strict inequality is obtained. Moreover, by choosing $\epsilon \ll 1$ arbitrarily small, this solution has an objective value that is arbitrarily close to the optimal objective value of the aforementioned relaxation. In other words, a slight perturbation of the solution to this problem gives a policy that achieves the planner's goal, while resulting in a cost level that is arbitrarily close to the optimal one. Motivated by this, in the second regime we refer to the solution of (P2) as the planner's optimal policy. We also note that $(\sqrt{\mathrm{P} 2})$ is a tractable semidefinite program.

\section{Application: Economic Activity in NYC}

We next apply our framework to NYC and discuss how the economic activity in different neighborhoods needs to be reduced so as to curb the spread of the Covid-19 epidemic at a minimum economic loss. In Section 3.1 we explain the data sources used for the construction of the primitives of our model, and in Section 3.2 and Section 3.3 we respectively report our findings in the large and small infection regimes.

\subsection{Data and Assumptions}

There are five main types of data that we use to determine the primitives of our model: (i) disease-specific parameters (e.g., transmission rates, clinical rates) for Covid-19 (Li et al. 2020b), (ii) the infection counts for NYC and neighboring counties (NYC Health, 2020; The New York Times, 2020), (iii) populations of different neighborhoods (US Census Bureau, 2010), (iv) level of economic activity in different NYC neighborhoods (US Census Bureau, 2017), (v) SafeGraph data that records individuals' movements (SafeGraph, 2020). We next explain how we construct the different primitives of our model using these data. 


\begin{tabular}{|c|c|c|c|c|c|}
\hline Parameter & $\beta$ & $\kappa$ & $\rho$ & $\alpha$ & $\gamma$ \\
\hline Median & 1.12 & 0.27 & 0.14 & 0.55 & 0.29 \\
\hline (95\% CIs $)$ & $(1.06,1.19)$ & $(0.25,0.30)$ & $(0.10,0.18)$ & $(0.46,0.62)$ & $(0.27,0.32)$ \\
\hline
\end{tabular}

Table 1: Disease-specific parameters (source: Li et al., 2020b)

Disease-specific parameters. We use the estimates provided in recent literature (in particular Li et al., 2020b) to calibrate the disease parameters governing the spread of COVID19. We assume that the (daily) transmission rate $\beta$ is 1.12 with $95 \%$ credible interval (CI): $(1.06,1.19)$. The latency period is 3.69 days $(3.30,3.96)$, implying an infectious rate of $\kappa \approx 0.27$ which captures how fast the exposed agents become infectious. The infectious period is 3.47 days $(3.15,3.73)$, implying a recovery rate of $\gamma \approx 0.29$. Not all infections cause severe symptoms and are reported. We set the clinical rate, which is assumed to be equal to the reporting rate, equal to $\rho=0.14(0.10,0.18)$ following the estimates in Li et al. (2020b). The unreported cases have a lower transmission rate, captured by a discount factor $\alpha=0.55(0.46,0.62)$, i.e., the transmission rate for such cases is given by $\alpha \beta \approx 0.62$. According to Li et al. (2020b), these parameter values imply an effective reproduction rate of $2.38(2.03,2.77)$, in the absence of interventions. Moreover, they are largely consistent with other available estimates in the literature (e.g., Wu et al., 2020; Li et al., 2020a; Ferretti et al. 2020). We report our results by focusing on the median values presented above. The values are summarized in Table 1 .

The natural birth/date rate is much smaller relative to the remaining parameters of the model. Thus, for our computational studies we set it equal to zero.

Nodes $(\mathcal{N})$. NYC reports confirmed Covid-19 cases at the ZIP Code Tabulation Area (ZCTA) level (NYC Health, 2020). Motivated by this, we define the nodes in our analysis as the different ZCTAs in the city. ZCTAs consist of census blocks (CB) and while in most instances the ZCTA code is the same as the ZIP Code for an area, there can be slight differences (see US Census Bureau, 2020). We are mainly interested in understanding how NYC can reduce economic activity in different ZCTAs so as to curb the spread of the epidemic. However, due to the spatial nature of the spread of the epidemic infected individuals from outside NYC can infect susceptible individuals in NYC. To capture this we define additional nodes that correspond to the counties neighboring NYC $]^{3}$ Specifically, we used the SafeGraph data (discussed below) to obtain the 10 counties that have the largest inflow/outflow of individuals to/from NYC. These 10 counties together with the NYC ZCTAs constitute the nodes of our network. The list of these counties is provided in the appendix; see Appendix A.

Populations $\left(\left\{N_{i}\right\}\right)$. In order to define the total population of each node, we use the 2010 Census data that specifies populations at the CB level (US Census Bureau, 2010). We

\footnotetext{
${ }^{3}$ Here we aggregate data at the county level, as outside NYC the infection data was provided at the county level (The New York Times, 2020).
} 
use these to construct the population of each node in our network. These populations are relevant as they are the primitives $\left(\left\{N_{i}\right\}\right)$ of our spatial epidemic model.

Sizes of compartments $\left(\left\{S_{i} / E_{i} / I_{i}^{c} / I_{i}^{s c} / R_{i}\right\}\right)$. In Section 3.2, we focus on the state of the epidemic in NYC (and neighboring counties) on April 18, 2020, and solve the planner's decision problem. Note that, on the aforementioned date, the total number of infections in NYC was nontrivial; hence, to study the planner's decision problem we used the formulation given in Section 2.2. Recall that this formulation is based on the size of each of the disease compartments. Thus, we need to estimate the sizes of these components on April 18. We next describe how we do so. In what follows, we refer to April 18 as the target date, and denote it by $T_{\star}$. We also focus on April 21 and April 15, and denote these dates respectively by $T_{+}$and $T_{-}$.

For the nodes of our network, we can extract the number of confirmed cases on a given date using the data provided (NYC Health, 2020; The New York Times, 2020). On the other hand, recent studies suggest that in addition to the confirmed cases, there is a large number of individuals who have the disease but do not show any symptoms. In fact, the antibody studies suggest that in NYC about $24.7 \%$ of the individuals may have experienced the disease (as of April 27) (CBSN, 2020) - a much larger number than the number of confirmed cases $4^{4}$

To bridge the gap between the confirmed cases and the total infections implied by the antibody studies, we define an identification rate. We first describe how this quantity and the compartment sizes are obtained for the ZCTAs in NYC, and we then briefly discuss the extension of the approach to the neighboring counties.

We assume that on $T_{\star} 24.7 \%$ of NYC have had the disease..$^{5}$ We also compute the total number of confirmed infections in NYC by $T_{\star}$ (at all locations) using the dataset in NYC Health (2020). Dividing the latter number by the former, we obtain an identification rate, which is the fraction of actual infections that become confirmed cases. We assume that the identification rate is the same in all neighborhoods.

The data in NYC Health (2020) includes the daily numbers of confirmed cases by ZCTA for NYC. We analyze the data under the assumption that individuals spend 3 days in the exposed compartment and 3 days in the infected compartment (see our discussion above on the disease-specific parameters). We focus on the cumulative number of confirmed cases on dates $T_{\star}$ and $T_{-}$, in each ZCTA, and interpret the difference as the number of active identified infections on $T_{\star}$. By dividing this quantity by our identification rate, we obtain the number of infections that are active in each ZCTA of NYC on $T_{\star}$. Note that these active infections may be clinical or subclinical; thus, the aforementioned quantity is $I_{i}^{c}+I_{i}^{s c}$ for ZCTA $i$. We use the clinical rate $\rho$ reported above to solve for $I_{i}^{c}$ and $I_{i}^{s c}$ (where $\rho=I_{i}^{c} /\left(I_{i}^{c}+I_{i}^{s c}\right)$ ) on $T_{\star}$.

Similarly, we focus on the cumulative number of confirmed cases in each ZCTA on $T_{\star}$ and $T_{+}$. Subtracting the former number from the latter one yields the number of new

\footnotetext{
${ }^{4}$ Similarly, Hortaçsu et al. (2020) estimate that 4\%-14\% of actual infections had been reported in the US up to March 16.

${ }^{5}$ The antibodies appear with some delay after the infection. According to Wölfel et al. (2020), half of the infected mild cases developed antibodies within a week of the onset of the symptoms, whereas the rest developed antibodies in the second week. Even though $24.7 \%$ was reported on April 27, due to the aforementioned delay as well as the time spent surveying the patients, it is reasonable to expect a similar infection rate earlier as well, consistent with our assumption on $T_{\star}$.
} 
infections by $T_{+}$. Before being identified as infected, these individuals were in the exposed compartment. Thus, this difference is interpreted as the number of exposed individuals on $T_{\star}$ who are eventually identified as infected. Dividing this quantity by the identification rate at each ZCTA, we obtain $\left\{E_{i}\right\}$.

Finally, all of the confirmed cases on $T_{-}$move to the recovered compartment by $T_{\star}$ (under the assumption that individuals spend 3 days in the diseased compartment). Dividing the relevant number at each ZCTA by the identification rate yields $\left\{R_{i}\right\}$ on $T_{\star}$.

Consider location $i$, and the compartment sizes $E_{i} / I_{i}^{c} / I_{i}^{s c} / R_{i}$ described above. We compute the difference between $N_{i}$ and the sum of these quantities to obtain the size of the susceptible compartment, $S_{i}$, for all ZCTAs $i$.

The compartment sizes for the 10 counties neighboring NYC are obtained following a similar approach. The only difference is that the antibody tests suggest lower prevalence in these counties than in NYC (CBSN, 2020). Thus, we replace the number $24.7 \%$ with a county-specific number and repeat the same process (using the data from The New York Times (2020) to compute the county-specific identification rates).

Baseline economic activity $\left\{c_{i}\right\}$. Longitudinal Employer-Household Dynamics (LODES) data (US Census Bureau, 2017) contains detailed information about the local economic activity. We use this dataset to obtain the number of employees in each ZCTA of NYC. We use the number of employees as a proxy for the baseline economic activity, and, in particular for a given ZTA $i$, we set $c_{i}$ to be the number of employees in this ZCTA. Thus, the problem of the planner can be alternatively formulated as the problem of curbing the spread of Covid-19 while incurring the minimum number of (temporary) job losses.

Edge weights $\left\{\tau_{i j}\right\}$. SafeGraph uses data from a number of mobile devices and applications to derive anonymized location data that provides insights into locations visited by individuals as well as their movements across different locations. To preserve anonymity, the data is aggregated at the census block group (CBG) level. More specifically, the SafeGraph dataset identifies a "home" CBG for each device/individual and reports the median home dwell time for each CBG. In addition, it reports the daily number of individuals who go from their home CBG to various destination CBGs. Similarly to ZCTAs, each CBG consists of a number of CBs. But, in general, CBGs neither contain nor are contained by ZCTAs. To get around this difficulty, we first disaggregate the SafeGraph data at the CB level. In particular, we focus on all "trips" that originate from a home CBG $i$ and that go to a destination CBG $j$. We assume that the fraction of these trips that originate from a particular CB in CBG $i$ is simply given by the ratio of the population of this CB (obtained from the data in US Census Bureau, 2010 to the total population of CBG $i$. Similarly, we assume that the fraction of these trips that end at a particular $\mathrm{CB}$ in $\mathrm{CBG} j$ is given by the ratio of the area of this CB to the area of CBG $j !^{6}$ Under this assumption, we can break down the "flow" of individuals between home/destination CBGs to the flow between home/destination CBs.

\footnotetext{
${ }^{6}$ Note that we distribute the origins according to the populations of the CBs whereas we distribute the destinations according to the areas. This is because, the number of "homes" in a CB is naturally proportional to the population of that $\mathrm{CB}$, and hence when allocating origins of trips to $\mathrm{CBs}$ it is natural to focus on populations. On the other hand, the destinations of the trips can be to nonresidential areas and focusing on populations in that case could be misleading. Therefore, in the latter case, we focus on the areas of CBs.
} 
Then we aggregate these quantities at the ZCTA level for NYC (county level for the nodes outside NYC) to define the flow of individuals from one node (ZCTA in NYC or a neighboring county) to another. Similarly we assume that all CBs in the same CBG share the same home dwell time, and we define the home dwell time for a ZCTA as the average of these for the CBs that are contained in the ZCTA (and similarly for the neighboring counties).

This construction allows us to obtain a representative home dwell time for each node. Moreover, it allows us to capture the number of trips (in the dataset) from one node to another. Given node $i$, we focus on the number of trips to a node $j$ divided by the total number of trips leaving $i$. We use this quantity to define the fraction of time the individuals from $i$ spend in $j$ in aggregate, i.e., the parameter $\tau_{i j}$ in our model. More precisely, if the home dwell time at node $i$ is $h_{i}$ hours, and the number of trips from $i$ to $\ell$ is $k_{i \ell}$, we let $\tau_{i j}=\left(1-\frac{h_{i}}{24}\right) \times \frac{k_{i j}}{\sum_{\ell} k_{i \ell}}$.

\subsection{Large Infection Regime (NYC)}

In this section, we explore the solutions of $(\overline{\mathrm{P} 1})$ for the problem primitives defined in the previous section. In both cases, we consider a planner who can control the economic activity at the nodes that correspond to the NYC ZCTAs. Note that these correspond to some of the nodes in our network, while the remaining nodes correspond to neighboring counties. We assume that the economic activity level in these counties is exogenously fixed at some level $y$. In our optimization formulations, we capture this by imposing an additional constraint $x_{i}=y$ for $i$ that corresponds to a neighboring county. We analyze different scenarios with $y \in\{0,0.4,0.8,1\}$ and discuss how the planner's optimal targeting solution changes depending on the activity level in the neighboring counties.

The optimal solutions for ( $(\mathrm{P} 1)$ and the three scenarios described above are depicted in Figure 1. In this as well as the subsequent figures, we shade the ZCTAs with different colors that capture the value the quantity of interest takes in each ZCTA. The color bar summarizes the mapping between the colors and the values. We also report the ratio of the optimal objective value to the total baseline economic value $\sum_{i} c_{i}$. We refer to this quantity as efficiency, and note that this quantity is less than or equal to one, and it is close to one only if the economic restrictions are very mild.

A number of important observations are worth pointing out, and we highlight these below (O1-O4).

Figure 1 shows that the optimal solution of ( $(\overrightarrow{\mathrm{P} 1})$ and the associated efficiency level are sensitive to the economic activity level in the neighboring counties. When the economic activity in the neighboring regions is completely suspended $(y=0)$, a targeted restriction policy can guarantee $48.8 \%$ efficiency, while achieving the policy goal of having reductions in the number of infections in all neighborhoods. On the other hand, when the economic activity level in the neighboring counties is $y=0.4,0.8$ or 1 , the efficiency reduces to $45.0 \%$, $40.0 \%$ and $37.2 \%$, respectively. In the last case, the economic activity almost completely ceases in Manhattan. Moreover, relative to the $y=0$ case, the reduction in the efficiency is $23.8 \%$. This observation highlights the necessity of coordination between states or other governing bodies to ensure low economic losses when achieving the policy goal of guaranteeing reductions in infections in all neighborhoods. 
$(P 1), y=0$, efficiency $=0.488$

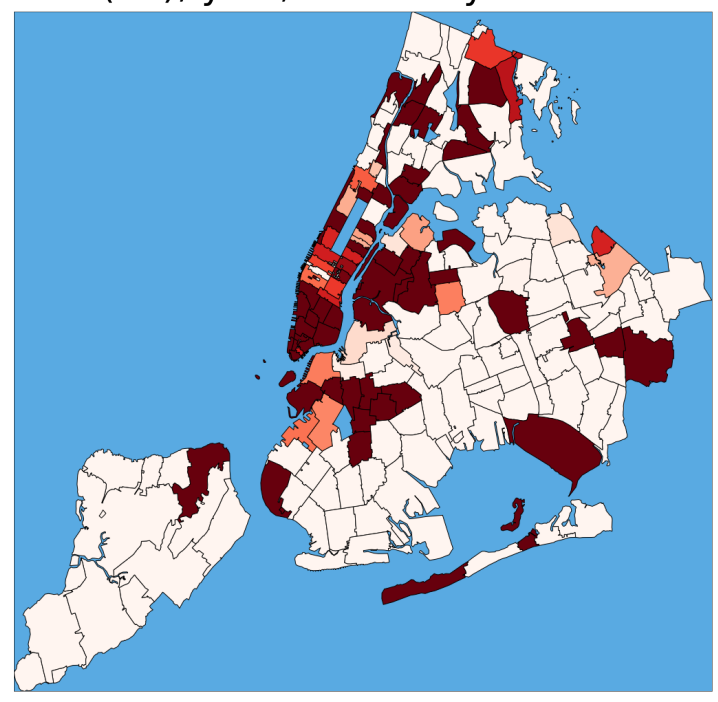

$(P 1), y=0.8$, efficiency $=0.400$

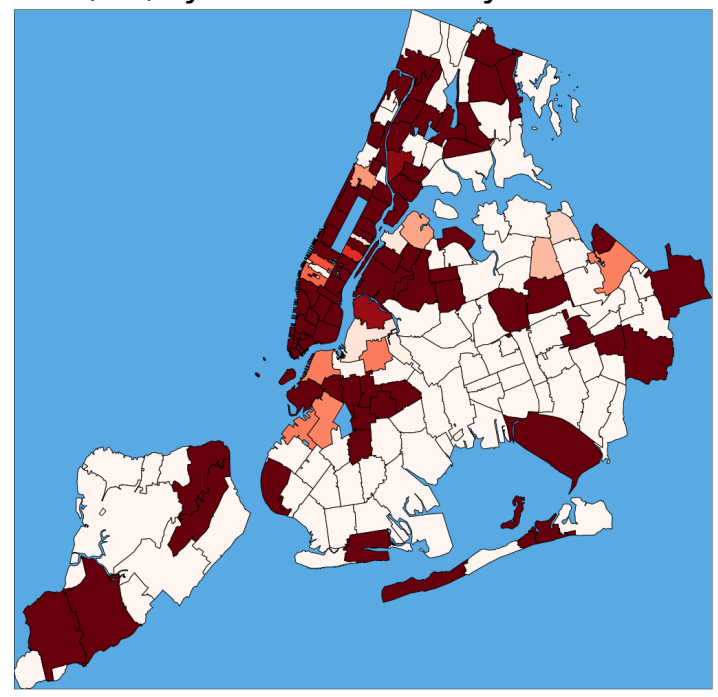

$(P 1), y=0.4$, efficiency $=0.450$

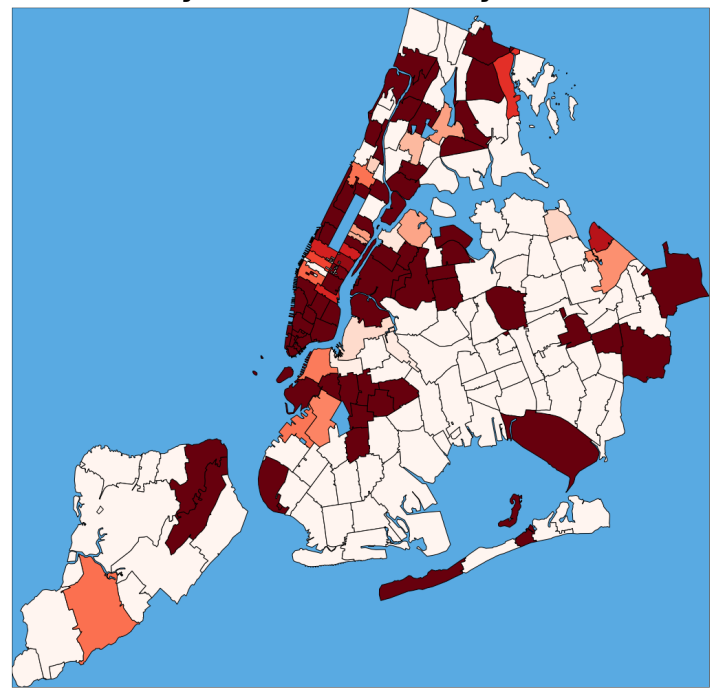

$(P 1), y=1$, efficiency $=0.372$

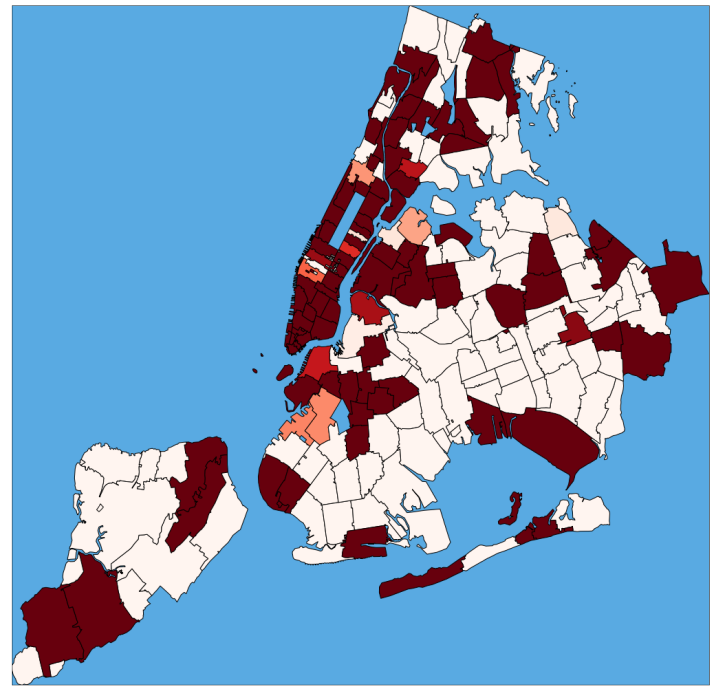

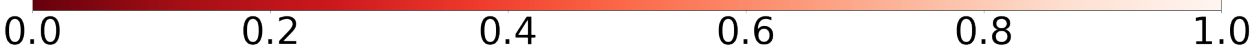

Figure 1: Optimal economic activity levels $\left\{x_{i}\right\}$ in different ZCTAs. 
O1: Coordination between governing bodies of different states/counties is necessary to limit economic losses while achieving the policy goals.

When the economic activity in the neighboring counties is relatively low, the optimal solution involves having some economic activity in Midtown Manhattan, while restricting economic activity substantially (and in some cases completely) almost elsewhere in Manhattan. This is partly due to the fact that the Midtown ZCTAs have the largest amount of baseline economic activity in the city (see Figure 6 in the appendix). It is worth noting that other neighborhoods, such as Lower Manhattan also have substantial baseline economic activity, yet the permitted economic activity at the optimal solution is zero. This is due to the fact that if the permitted economic activity in those neighborhoods was increased, then due to the spatial nature of the epidemic, in order to achieve the policy goals the economic activity level in other locations (such as Midtown) would need to be reduced. The cost of the latter reduction outweighs the benefit of increasing economic activity in Lower Manhattan.

O2: In Manhattan, some economic activity is permitted in Midtown, driven by the economic value of this location. To achieve the policy goal, the impact of this location is balanced by severely restricting economic activity almost elsewhere in Manhattan.

It is worth highlighting that the restrictions can change drastically even between adjacent neighborhoods (e.g., those in Staten Island). More interestingly, even among neighborhoods with similar baseline economic activity levels, the ones with higher levels of permitted economic activity, are not necessarily those with lower levels of infection. For instance, in Staten Island, the neighborhoods that are permitted to continue economic activity have higher infection rates than the adjacent neighborhoods that are completely shut down (see Figure 7 and Figure 8 in the appendix). This is because in deciding how much economic activity to allow, in addition to location-specific metrics (such as the infection rate in a neighborhood), the spillovers across neighborhoods also matter (i.e., whether allowing economic activity in a neighborhood will import/export the disease to other neighborhoods substantially).

O3: The spatial aspect of disease spread and spillovers across neighborhoods plays a key role in deciding where to resume economic activity.

Note that the targeted policies obtained from the solution of (P1) suggest drastic differences in permitted economic activity levels even in adjacent ZCTAs (e.g., with one ZCTA having full economic activity and the other one having zero). This may be undesirable due to fairness concerns. A conceptually more fair alternative is to permit the same level of economic activity in all neighborhoods. The fairness requirement restricts the policies that the planner can use and reduces efficiency. How does the efficiency of the optimal policy change due to this requirement?

To analyze this, we resolve $(\mathrm{P} 1)$ by imposing the additional constraint that $x_{i}=x_{j}$ for $i, j$ that correspond to the ZCTAs in NYC. In Figure 2, we show the efficiency in the optimal solutions of this LP as well as the original one for different values of the permitted economic activity $y$ in the neighboring counties. 


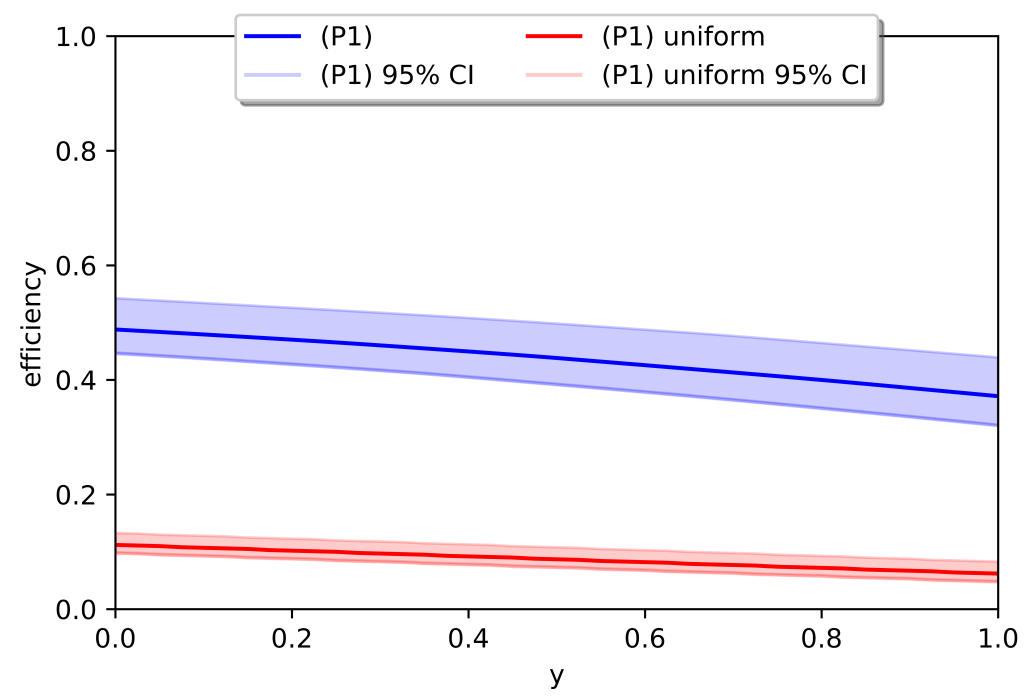

Figure 2: Efficiency vs. permitted economic activity $y$ in the neighboring counties.

In this figure, we use solid lines to illustrate the efficiency levels obtained with the optimal targeted/uniform policies when the disease parameters take their median values reported in Table 1. In addition, we fit Weibull distributions to the median values and the $95 \%$ CIs (see Table 1) of these parameters. We then sample the relevant parameters from the corresponding distributions and create 100 problem instances. In each of these problem instances we obtain optimal policies as well as the corresponding efficiency levels (for different $y$ ). In Figure 2 we shade the $95 \%$ CI associated with the induced optimal efficiency levels under the two policies. The results for the $95 \%$ CI are consistent with those associated with the median parameter values, thereby indicating the robustness of our conclusions to the exact values of the disease parameters.

Figure 2 establishes that there are drastic gains to targeting different neighborhoods under the policy goal of reducing infections in all neighborhoods. The gains due to targeting are large (they are roughly fourfold or more where the exact multiplier depends on $y$ ).

\section{O4: Targeting achieves policy goals at a much lower economic cost.}

\subsection{Small Infection Regime (NYC)}

We next analyze the asymptotic stability of the disease-free equilibrium in NYC. To this end, we assume that all of the model primitives but the compartment sizes are as given in Section 3.1. Since our focus is on the disease-free equilibrium, we assume that initially all of the agents are in the susceptible compartment. As discussed earlier, asymptotic stability is equivalent to having $\mathscr{R}_{e}<1$ and qualitatively it guarantees that if a small number of infections are introduced to the population, the infections will disappear without a large number of individuals getting infected. 
As in Section 3.2, we focus on the problem of the planner who can control economic activity at the nodes that correspond to the NYC ZCTAs. For the remaining nodes (i.e., in counties neighboring $\mathrm{NYC}$ ), we assume that the economic activity level is exogenously set equal to $y \in\{0,0.4,0.8\}$. Observe that this boils down to formulating (P2) with the additional case $x_{i}=y$ for any node $i$ that is not a ZCTA of NYC. We note that for $y \geq 0.85$ the planner's problem becomes infeasible. That is, if the neighboring counties have $85 \%$ or more economic activity, due to the infections that take place there regardless of the policy NYC follows, then the infections could impact a substantial number of agents in the system and the disease-free equilibrium would not be stable.

We show the optimal targeted reduction in the economic activity levels in Figure 3, where we also report the efficiency of the solution. The results suggest that the solutions and the optimal objectives of the first two scenarios are fairly close. This suggests that in the regime where the permitted economic activity in the neighboring counties is inherently small (i.e., $y \leq 0.6$ ), the impact of these counties on NYC is also relatively small. Hence, the optimal solution is relatively insensitive to the exact value of $y$ (for as long as it remains small).

The optimal policy of the planner (in all three scenarios) involves permitting almost full economic activity in some regions, e.g., a few ZCTAs in Midtown Manhattan, Lower Manhattan, and the airports.7 Note that these locations are places where many employees from different neighborhoods mix together. So, arguably, the infections here could quickly spread and trigger a contagion. On the other hand, these locations are also very important economically. In particular, Figure 6 in the appendix shows that these locations also have substantially higher baseline economic activity levels than the remaining locations. Thus, it is optimal for the planner to keep the economic activity continuing at these locations as much as possible, while suspending or reducing the economic activity elsewhere.

It is possible to obtain further intuition on the optimal solution by focusing on another metric: the inflow-to-economic-activity ratio. For neighborhood $i$ this metric is obtained by dividing the total amount of time individuals from different neighborhoods spend at node $i$ (given by $\sum_{j} \tau_{j i} N_{j}$ ) by the baseline economic activity level $c_{i}$. Intuitively, when this ratio is small either the mixing (and hence the infection risk) in a node is small, or the economic value of the relevant node is large. In either case, the planner should benefit from not restricting the economic activity in the aforementioned neighborhoods too much. Figure 9 in the appendix suggests that this intuition has merit (despite the aforementioned metric not capturing important features of the disease dynamics): the neighborhoods for which this metric takes smaller values also experience limited reduction in the economic activity according to the optimal solution given in Figure 3.

The type of targeted restriction of the economic activity described above may not be ideal due to fairness concerns. We next explore how the optimal solution changes, if we, in addition, we require the economic restrictions to apply uniformly to all the regions. This corresponds to imposing the additional constraint $x_{i}=x_{j}$ for $i, j$ that correspond to the ZCTAs of NYC in the optimization formulation ( $\sqrt{\mathrm{P} 2}$. In Figure 4, we plot the efficiency under the optimal targeted vs. uniform policies (obtained by solving the relevant variants of

\footnotetext{
${ }^{7}$ Here the result about the airports should be interpreted cautiously, since we did not model diseases "imported" due to incoming flights and accounting for them could change the optimal activity levels at those locations.
} 
(P2), $y=0$, efficiency $=0.930$

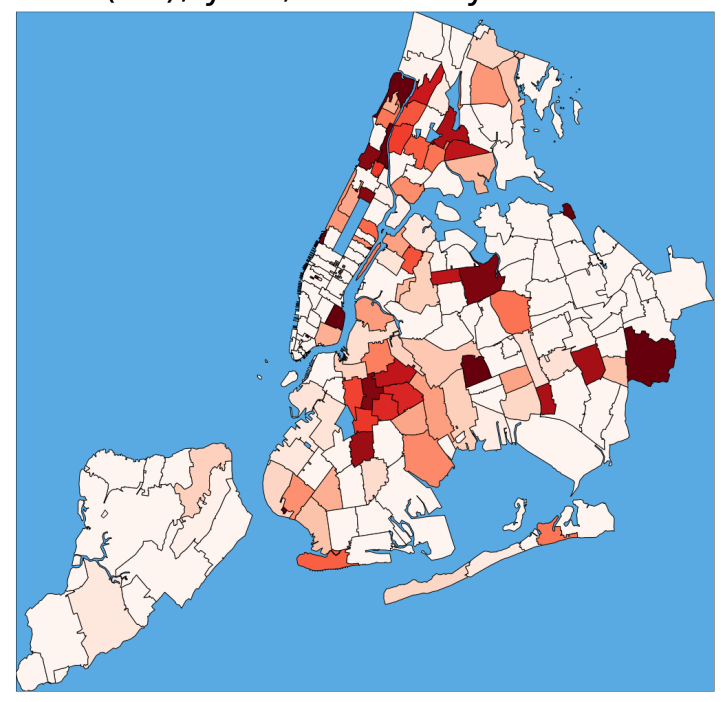

$(P 2), y=0.8$, efficiency $=0.874$
(P2), $y=0.4$, efficiency $=0.924$

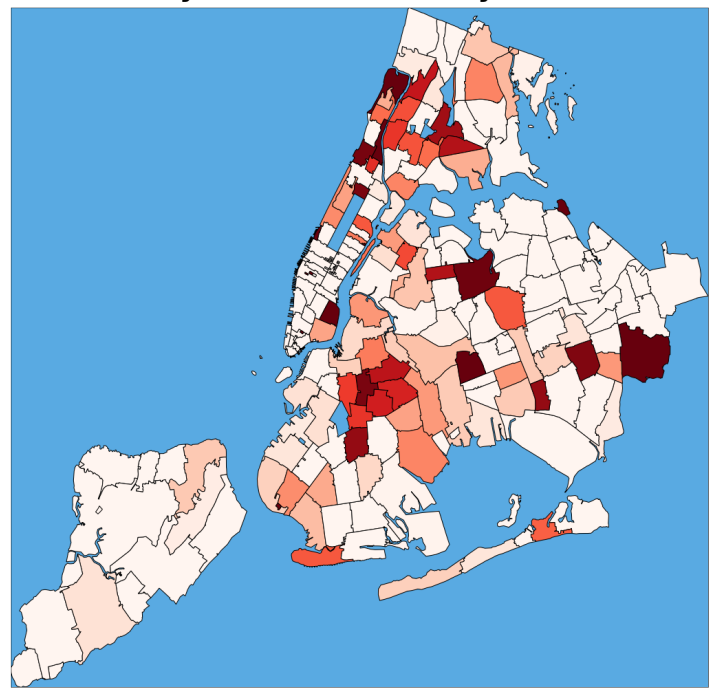

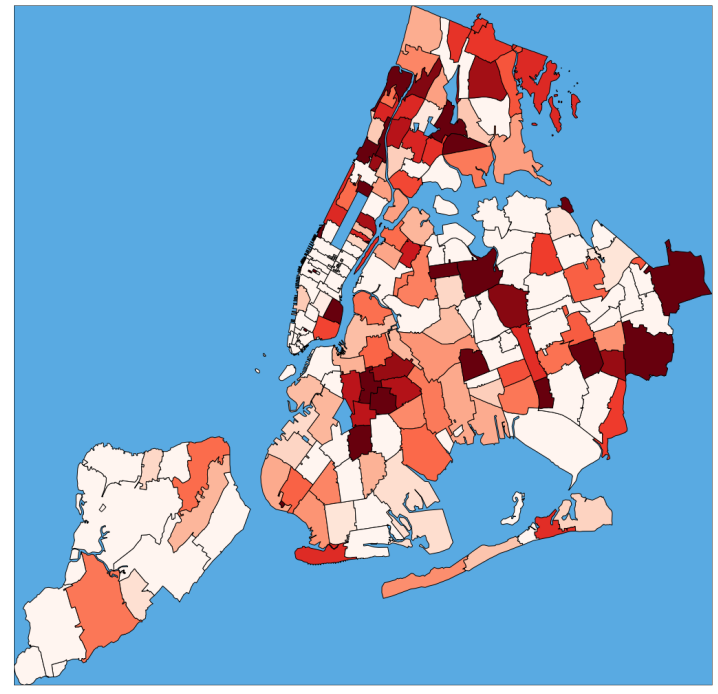

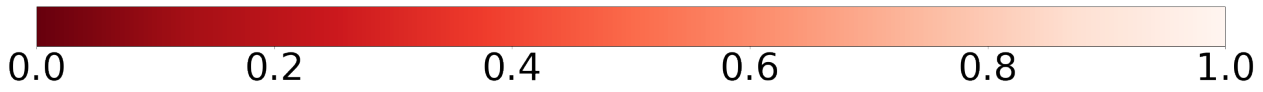

Figure 3: Optimal economic activity levels $\left\{x_{i}\right\}$ in different ZCTAs. 


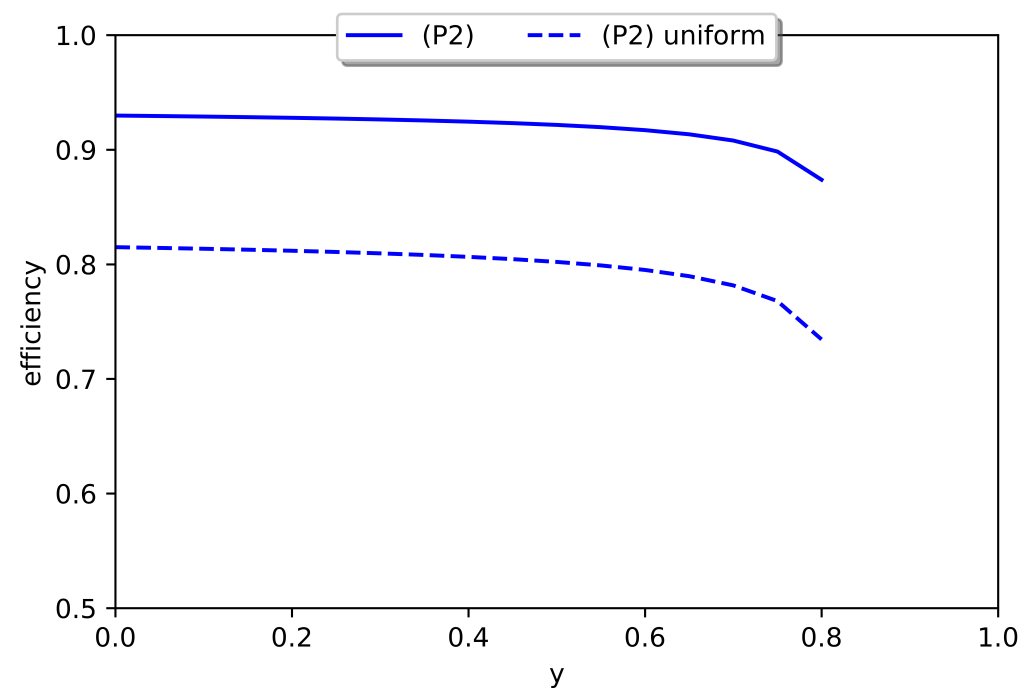

Figure 4: Efficiency vs. permitted economic activity $y$ in the neighboring counties.

$(\bar{P} 2)$ as a function of the economic activity level $y$ in the neighboring counties. This figure establishes that the reduction in the efficiency due to using uniform policies is about $14 \%$. Moreover, neither objective value seems very sensitive to small $y$, as observed earlier. Our findings highlight the value of targeting different neighborhoods in efforts keep $\mathscr{R}_{e}$ below 1 .

\section{Concluding Remarks}

In this paper, we focus on a spatial model of epidemic spread, and investigate the problem of a planner who can reduce the economic activity in different neighborhoods in a targeted way in order to curb the spread of an epidemic. The intervention is costly, and optimal targeting policies are discussed. The problem is relevant both in the regime where there is a large number of infections in society and in the regime where only a few agents are infected. In the first regime, we focus on the policy goal of reducing infections in each neighborhood or in aggregate (while minimizing the induced economic cost), and, in the second regime, we focus on ensuring that the small number of initial infections do not trigger an epidemic. We provide tractable convex optimization formulations of the planner's problem. We then use these formulations to explore the planner's optimal policies in fighting the Covid-19 epidemic in NYC.

Our analysis of NYC reveals a number of important insights, some of which we highlight here. First, targeting can enable achieving policy goals while ensuring a substantial reduction in economic losses relative to uniform shutdown policies. Second, under various scenarios, the planner finds it optimal to continue limited economic activity in Midtown neighborhoods, while almost completely suspending economic activity almost elsewhere in Manhattan. The reason for this is the significant economic value of the Midtown neighborhoods, and the planner finds it optimal to trade off the economic activity in this region with closures elsewhere in the city. Third, contrary to naive intuition, the neighborhoods 
where the economic activity should be reduced the most are not those with the highest infection rates: even among adjacent neighborhoods with similar economic values, it may be optimal to resume activity at those with higher infection rates depending on the structure of the spatial spread patterns between these neighborhoods and the others. Fourth, in the small infection regime, in order to achieve policy goals different governing bodies need to coordinate closures. For instance, if NJ counties resume economic activities it may not be possible for NYC to prevent an epidemic even with drastic closure decisions. Finally, when there are a few infected agents, a relatively weaker reduction in economic activity may be needed in order to ensure that $\mathscr{R}_{e}<1$ and that the disease does not impact a large number of individuals.

While we have striven to build a model consistent with epidemic, economic, and social activity data, we made several assumptions that affect these results. We made a uniformity assumption that restrictions on economic activity in a location would have the same relative impact on social activity and inter-regional movement across all locations. If this is not true (e.g., if Midtown economic activity has less of an effect on movement than other neighborhoods's economic activity), then many of the results may again be different. Finally, it is conceivable that the economic activity in some neighborhoods is more amenable to remote work. Thus, business closure may have less of an economic impact in these neighborhoods than in others. Such heterogeneity across different neighborhoods is not modeled in the paper, and could impact the optimal decisions of the planner.

This work opens up a number of interesting future directions. First, our focus has been on myopic and static policies that a planner can use to achieve some policy goals at the lowest possible costs. Such policies can be used to develop heuristic policies that control the epidemic over time as well, e.g., by resolving the static problems over time to adjust the interventions as the state of the epidemic evolves. Perhaps the most interesting (and challenging) direction is to study truly dynamic policies (as opposed to heuristic ones) that minimize total economic costs (over time and across neighborhoods) and that ensure a longterm policy goal (e.g., ensuring that the infection numbers will be below a threshold by a deadline). Such policies require carefully modeling the trajectory of the disease dynamics, and induce nontrivial optimal control problems that have a spatial as well as a temporal dimension. While the study of such dynamic epidemic control problems is challenging, it also appears quite timely and potentially impactful. Second, for Covid-19 there is still uncertainty about a number of disease-related parameters. A closer study of these as well as their impact on optimal closure decisions is needed. Third, in settings where there is uncertainty about the underlying parameters, the question of testing becomes important. How should the decision maker prioritize testing to collect information about the uncertain parameters and how could this be used to improve the overall objective? Fourth, other economic and social costs (other than employment numbers) are of interest to study. For instance, it is often argued that economic losses experienced by individuals can trigger loss of life, which could be comparable to (or more than) the deaths due to the epidemic. It is of interest to develop policies that minimize the total deaths by taking appropriate interventions. Finally, the proposed optimization framework for studying spatial epidemic models seems to be novel in the literature, and its applications to other problems in epidemiology could be of interest. 


\section{A Counties with largest trip rates to NYC}

\begin{tabular}{|cccc|}
\hline & Fairfield County, CT & Bergen County, NJ & \\
Essex County, NJ & Hudson County, NJ & Middlesex County, NJ & Monmouth County, NJ \\
Union County, NJ & Nassau County, NY & Suffolk County, NY & Westchester County, NY \\
\hline
\end{tabular}

Table 2: Counties/nodes outside NYC

\section{B Additional NYC data}

In this section, we provide additional data on various quantities related to our problem. In particular, Figure 5illustrates the population in different neighborhoods and Figure 6 shows the number of employees. Figure 7 captures the relative number of infections in different neighborhoods $\left(I_{i}^{c}+I_{i}^{s c}\right)$ on $T_{\star}$, and Figure 8 shows the same quantity after dividing it by the population of the relevant neighborhood (i.e., $I_{i}^{c}+I_{i}^{s c}$ divided by the population of $i$ ). All of the figures show the normalized data; i.e., the value associated with each neighborhood is divided by the maximum value associated with a neighborhood.

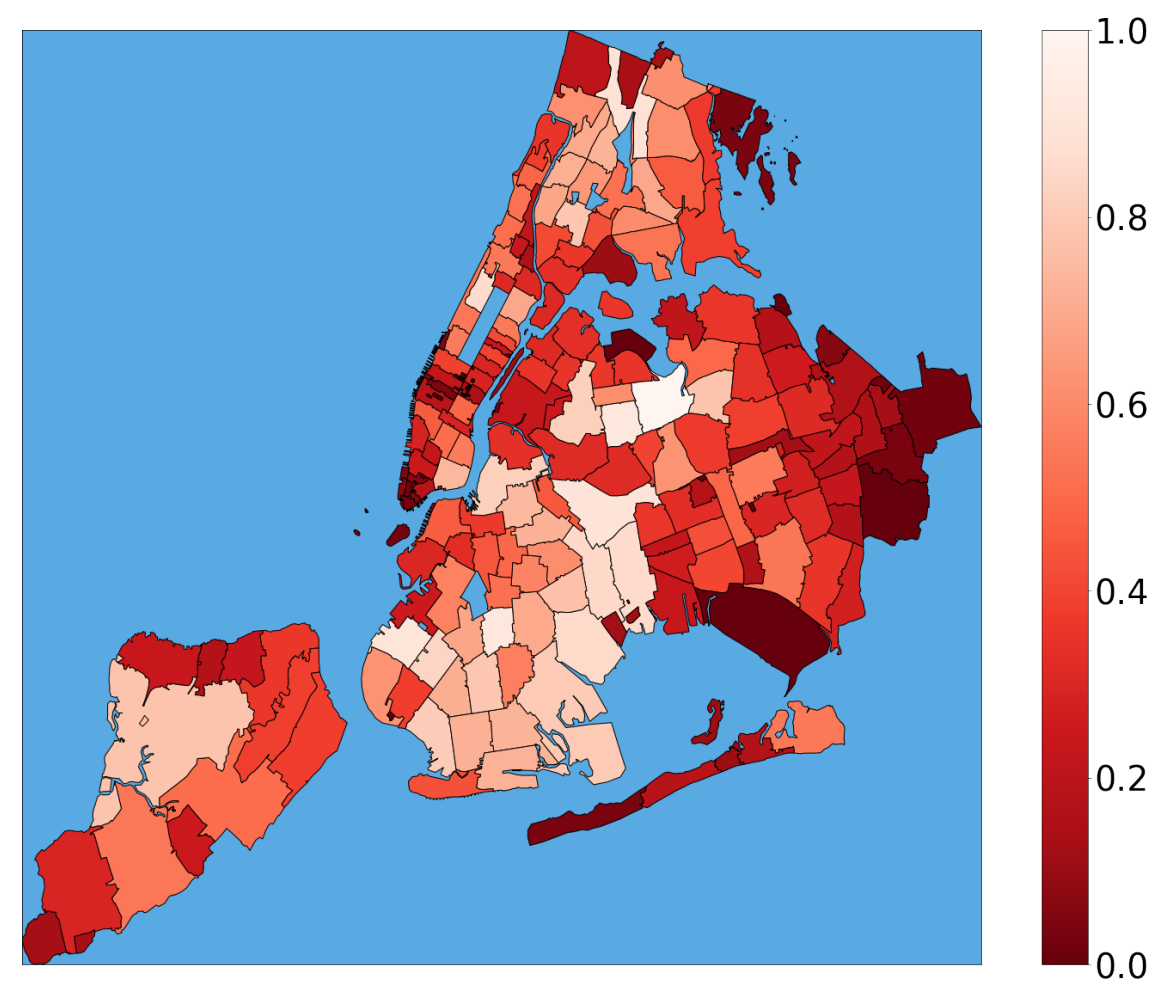

Figure 5: Normalized populations

Finally, we include another plot that seems to be closely related to our optimal solutions. Consider the total amount of time individuals from different neighborhoods spend at node 

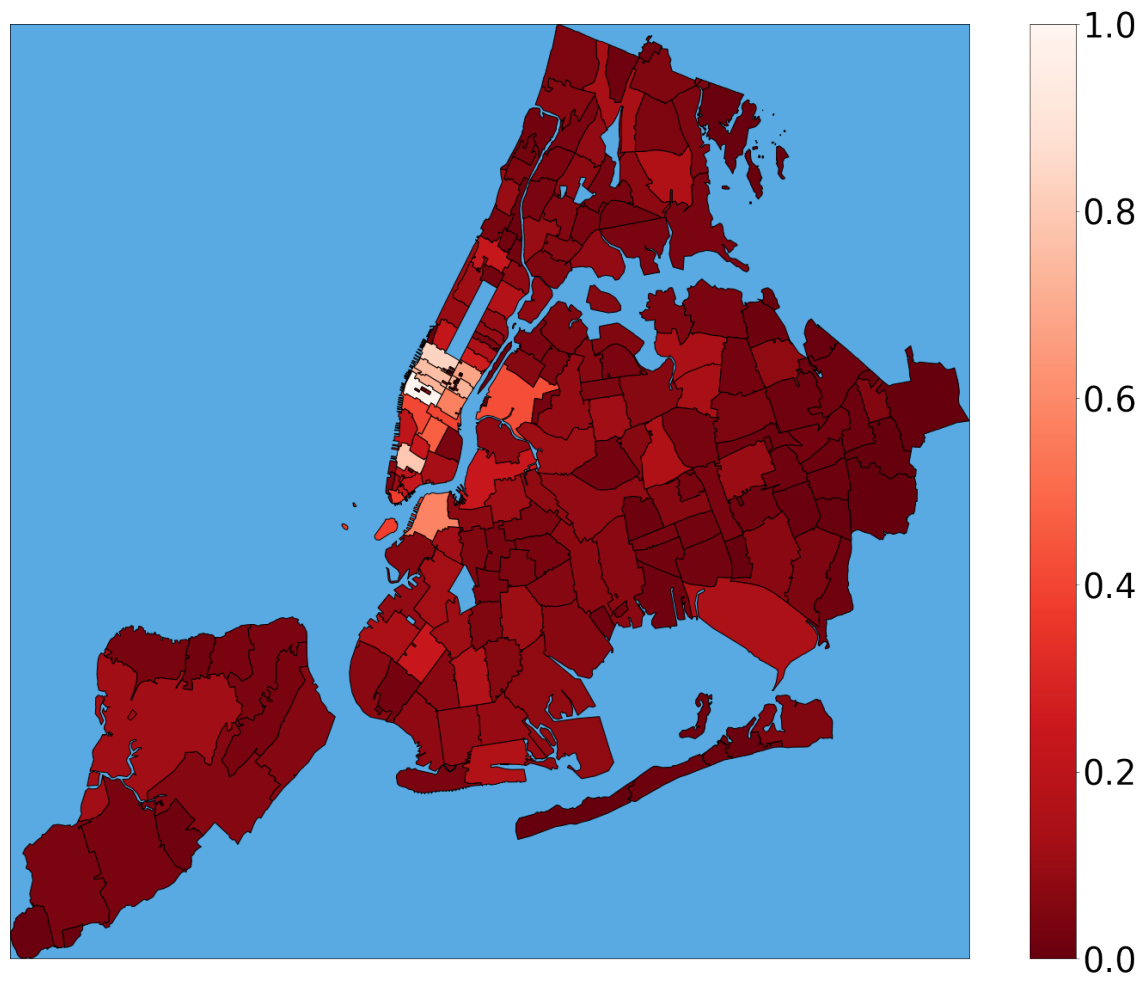

Figure 6: Normalized employment numbers
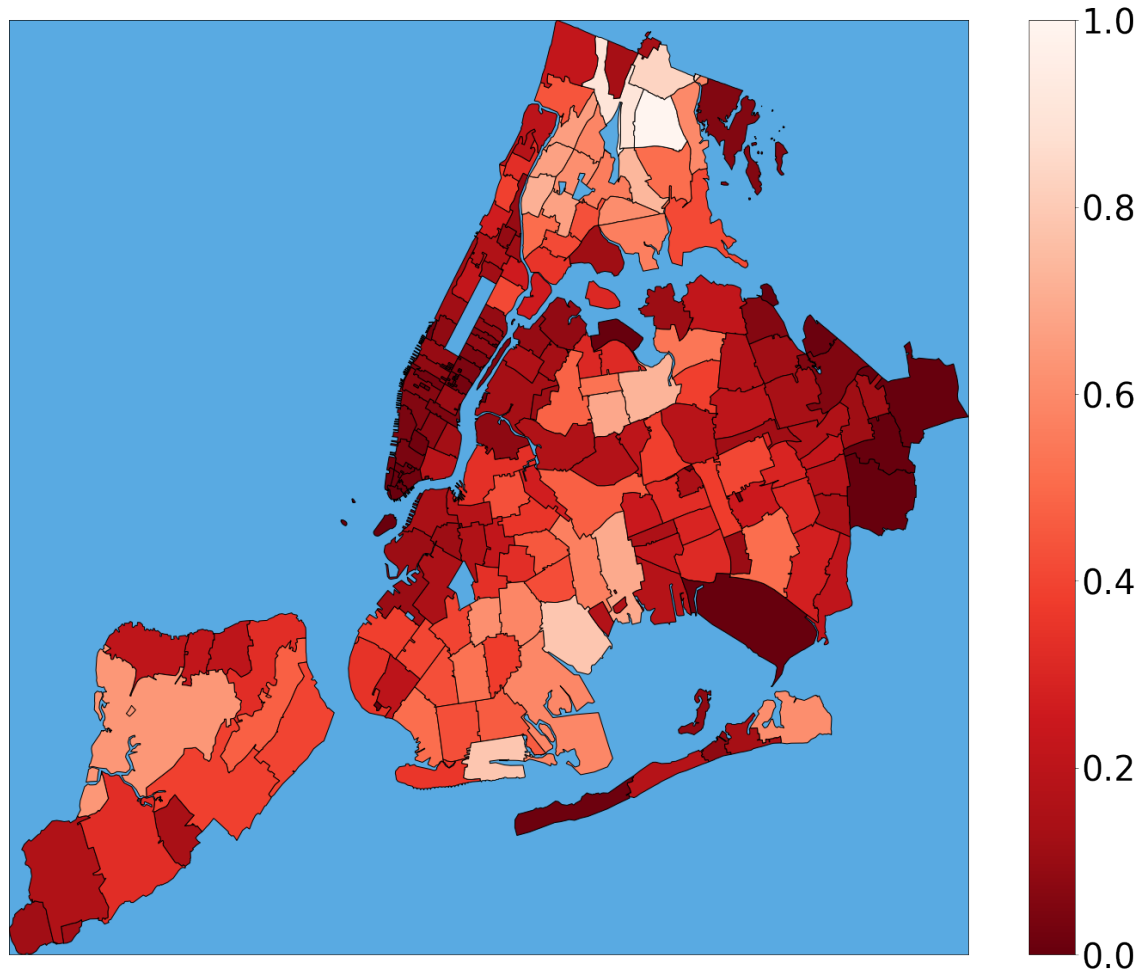

Figure 7: Normalized infection numbers 


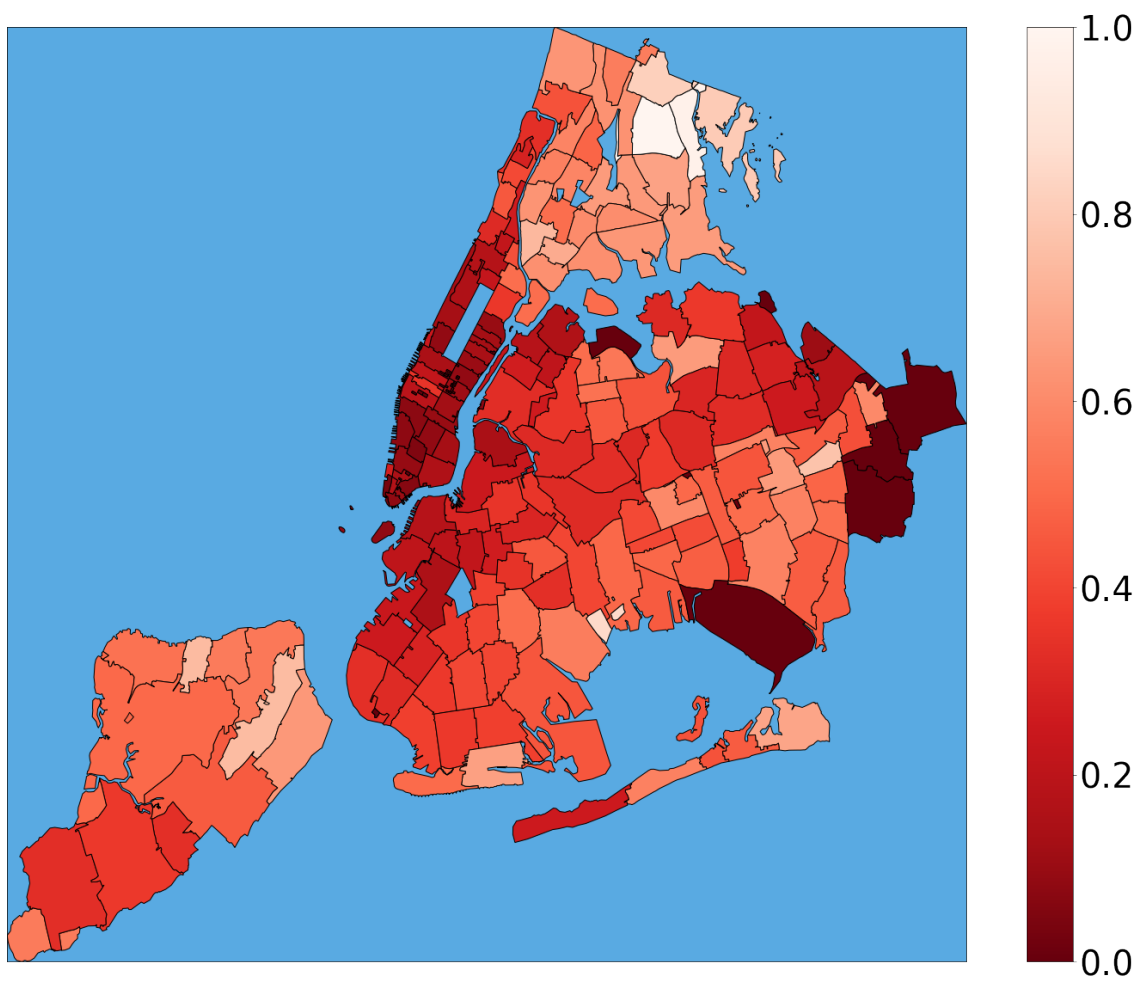

Figure 8: Normalized infection-to-population ratio

$i$ and divide this quantity by the baseline economic activity $c_{i}$, i.e., the inflow-to-economicactivity ratio. $\sum_{j} N_{j} \tau_{j i} / c_{i}$. This quantity, after normalization, is plotted in Figure 9 .

The inflow-to-economic-activity ratio for neighborhood $i$ is large, when the numerator is large or the denominator is small. In the first case, $i$ is a neighborhood where many agents (possibly from different neighborhoods) mix. This can trigger the spread of the epidemic. In the second case, the baseline economic activity in $i$ is small, and hence the planner can reduce economic activity without suffering a large loss. Thus, intuitively the planner benefits from restricting economic activity when this ratio is large. While this crude metric misses a number of important features of the epidemic spread process (e.g., the rates at which individuals move between compartments), as discussed in Section 3 in some cases it is aligned relatively well with our optimal solutions: the neighborhoods where this ratio is the smallest (largest) often see larger (smaller) levels of permitted economic activity under the planner's policy.

\section{References}

Jason Abaluck, Judith A. Chevalier, Nicholas A. Christakis, Howard Paul Forman, Edward H. Kaplan, Albert Ko, and Sten H. Vermund. The case for universal cloth mask adoption and policies to increase supply of medical masks for health workers. Working paper, SSRN, 2020. URL https://ssrn. com/abstract=3567438.

Daron Acemoglu, Victor Chernozhukov, Iván Werning, and Michael D Whinston. A multi- 


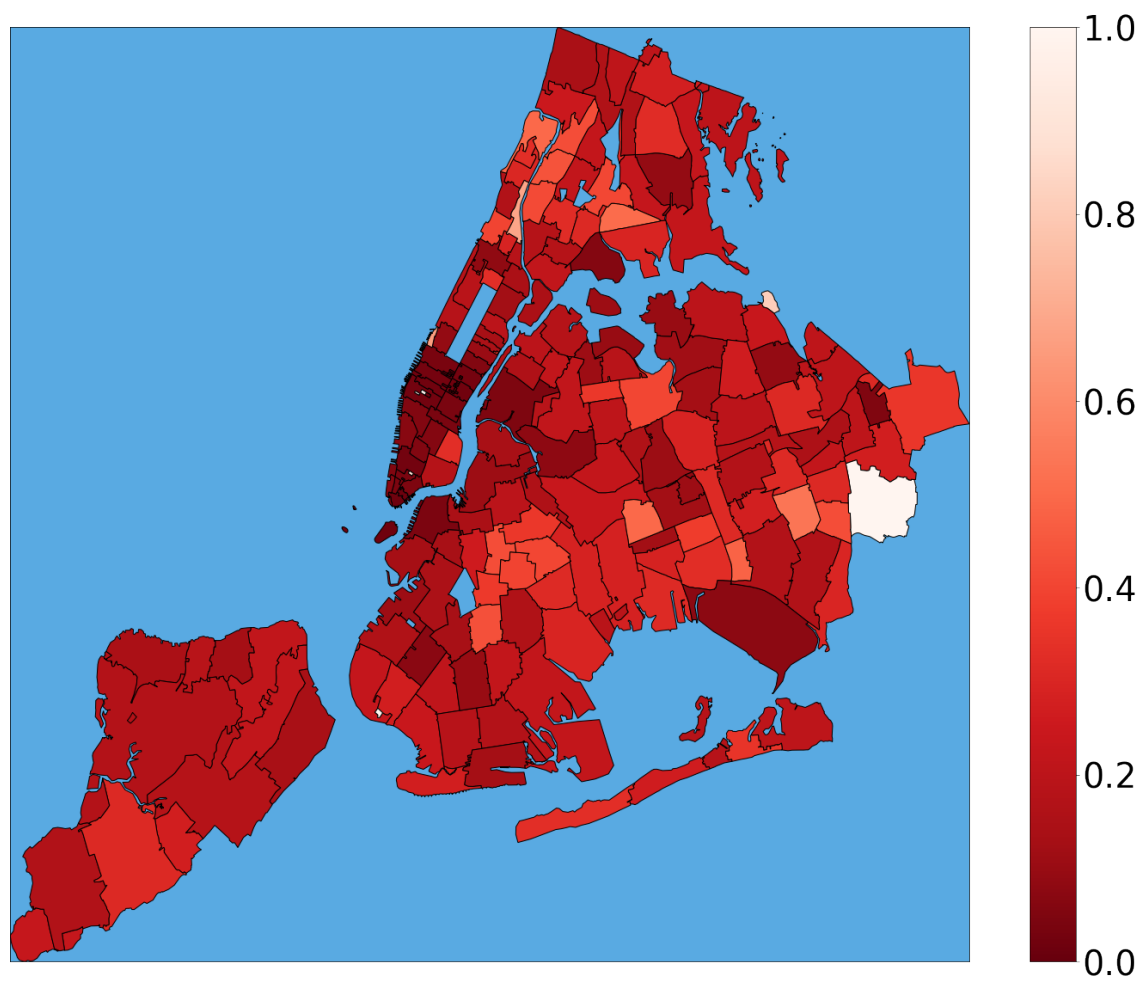

Figure 9: Inflow-to-economic-activity ratio

risk sir model with optimally targeted lockdown. Working Paper 27102, National Bureau of Economic Research, May 2020. URL http://www.nber.org/papers/w27102.

Linda JS Allen, Fred Brauer, Pauline Van den Driessche, and Jianhong Wu. Mathematical epidemiology, volume 1945. Springer, 2008.

Fernando E Alvarez, David Argente, and Francesco Lippi. A simple planning problem for covid-19 lockdown. Working Paper 26981, National Bureau of Economic Research, April 2020. URL http://www. nber .org/papers/w26981.

Roy M Anderson, B Anderson, and Robert M May. Infectious diseases of humans: dynamics and control. Oxford university press, 1992.

Scott R Baker, Nicholas Bloom, Steven J Davis, Kyle Kost, Marco Sammon, and Tasaneeya Viratyosin. The unprecedented stock market reaction to covid-19. Covid Economics: Vetted and Real-Time Papers, 1(3), 2020a.

Scott R Baker, Nicholas Bloom, Steven J Davis, and Stephen J Terry. Covid-induced economic uncertainty. Technical report, National Bureau of Economic Research, 2020b.

Scott R Baker, Robert A Farrokhnia, Steffen Meyer, Michaela Pagel, and Constantine Yannelis. How does household spending respond to an epidemic? consumption during the 2020 covid-19 pandemic. Technical report, National Bureau of Economic Research, 2020c. 
BNO. Tracking coronavirus: Map, data and timeline. https://bnonews.com/index.php/ 2020/04/the-latest-coronavirus-cases/, 2020.

Fred Brauer, Carlos Castillo-Chavez, and Zhilan Feng. Mathematical Models in Epidemiology. Springer, 2019.

Eric Budish, Anil Kashyap, Ralph Koijen, and Brent Neiman. Three pillars of the economic policy response to the covid-19 crisis. Policy notes, University of Chicago, Becker Friedman Institute for Economics Working Paper, 2020. URL https://faculty.chicagobooth. edu/eric.budish/research/IGM-Covid19-EconomicPolicyResponse.pdf.

Eric B Budish. R i 1 as an economic constraint: Can we 'expand the frontier' in the fight against covid-19? University of Chicago, Becker Friedman Institute for Economics Working Paper, (2020-31), 2020.

CBSN. Coronavirus antibodies present in nearly $25 \%$ of all nyc residents, cuomo says; un-pause in certain regions of ny might begin in may. https : / / newyork . cbslocal . com / 2020 / 04 / 27 / coronavirus-antibodies-present-in-nearly-25-of-all-nyc-residents/, 2020.

Matteo Chinazzi, Jessica T Davis, Marco Ajelli, Corrado Gioannini, Maria Litvinova, Stefano Merler, Ana Pastore y Piontti, Kunpeng Mu, Luca Rossi, Kaiyuan Sun, et al. The effect of travel restrictions on the spread of the 2019 novel coronavirus (covid-19) outbreak. Science, 368(6489):395-400, 2020.

Olivier Coibion, Yuriy Gorodnichenko, and Michael Weber. Labor markets during the covid19 crisis: A preliminary view. Technical report, National Bureau of Economic Research, 2020.

Odo Diekmann, Johan Andre Peter Heesterbeek, and Johan AJ Metz. On the definition and the computation of the basic reproduction ratio $\mathrm{r} 0$ in models for infectious diseases in heterogeneous populations. Journal of mathematical biology, 28(4):365-382, 1990.

Kimon Drakopoulos and Ramandeep S. Randhawa. Why perfect tests may not be worth waiting for: Information as a commodity. Working paper, SSRN, 2020. URL https: //ssrn. com/abstract=3565245.

Kimon Drakopoulos and Fanyin Zheng. Network effects in contagion processes: Identification and control. Columbia Business School Research Paper, (18-8), 2017.

Kimon Drakopoulos, Asuman Ozdaglar, and John N Tsitsiklis. An efficient curing policy for epidemics on graphs. IEEE Transactions on Network Science and Engineering, 1(2): $67-75,2014$.

Kimon Drakopoulos, Asuman Ozdaglar, and John N Tsitsiklis. When is a network epidemic hard to eliminate? Mathematics of Operations Research, 42(1):1-14, 2017. 
Luca Ferretti, Chris Wymant, Michelle Kendall, Lele Zhao, Anel Nurtay, Lucie AbelerDörner, Michael Parker, David Bonsall, and Christophe Fraser. Quantifying sars-cov-2 transmission suggests epidemic control with digital contact tracing. Science, 2020.

David Gershon, Alexander Lipton, and Hagai Levine. Managing covid-19 pandemic without destructing the economy. arXiv preprint arXiv:2004.10324, 2020.

A Glover, J Heathcote, D Krueger, and Rios-Rull J. Health versus wealth: On the distributional effects of controlling a pandemic. Working paper, Centre for Economic Policy Research, 2020. URL https://cepr.org/active/publications/discussion_papers/ dp.php?dpno=14606.

Niels Joachim Gormsen and Ralph SJ Koijen. Coronavirus: Impact on stock prices and growth expectations. University of Chicago, Becker Friedman Institute for Economics Working Paper, (2020-22), 2020.

Veronica Guerrieri, Guido Lorenzoni, Ludwig Straub, and Iván Werning. Macroeconomic implications of covid-19: Can negative supply shocks cause demand shortages? Technical report, National Bureau of Economic Research, 2020.

Samuel G. Hanson, Jeremy C. Stein, Adi Sunderam, and Eric Zwick. Business continuity insurance and loans: Keeping america's lights on during the pandemic. University of Chicago, Becker Friedman Institute for Economics Working Paper, 2020.

Zhiguo He and bibo Liu. Dealing with a liquidity crisis: Economic and financial policies in china during the coronavirus outbreak. University of Chicago, Becker Friedman Institute for Economics Working Paper, 2020.

Herbert W Hethcote. Qualitative analyses of communicable disease models. Mathematical Biosciences, 28(3-4):335-356, 1976.

Herbert W Hethcote and James W Van Ark. Epidemiological models for heterogeneous populations: proportionate mixing, parameter estimation, and immunization programs. Mathematical Biosciences, 84(1):85-118, 1987.

Ali Hortaçsu, Jiarui Liu, and Timothy Schwieg. Estimating the fraction of unreported infections in epidemics with a known epicenter: an application to covid-19. University of Chicago, Becker Friedman Institute for Economics Working Paper, (2020-37), 2020.

IMFBlog. The great lockdown: Worst economic downturn since the great depression. https : / / blogs . imf . org / 2020 / 04 / 14 / the-great-lockdown-worst-economic-downturn-since-the-great-depression/, 2020 .

Matthew O Jackson and Dunia López-Pintado. Diffusion and contagion in networks with heterogeneous agents and homophily. Network Science, 1(1):49-67, 2013.

Jayson S Jia, Xin Lu, Yun Yuan, Ge Xu, Jianmin Jia, and Nicholas A Christakis. Population flow drives spatio-temporal distribution of covid-19 in china. Nature, pages 1-11, 2020. 
Edward H Kaplan. Containing 2019-ncov (wuhan) coronavirus. Health Care Management Science, pages 1-4, 2020a.

Edward H. Kaplan. Covid-19 scratch models to support local decisions. Forthcoming, manufacturing \& services operations management, SSRN, 2020b. URL https : //ssrn.com/abstract $=3577867$.

Keystone. Coronavirus city and county non-pharmaceutical intervention rollout date dataset. https : / / www . keystonestrategy . com / coronavirus-covid19-intervention-dataset-model/, 2020.

Hassan K Khalil. Nonlinear systems; 3rd ed. Prentice-Hall, Upper Saddle River, NJ, 2002. URL https : //cds.cern.ch/record/1173048. The book can be consulted by contacting: PH-AID: Wallet, Lionel.

Qun Li, Xuhua Guan, Peng Wu, Xiaoye Wang, Lei Zhou, Yeqing Tong, Ruiqi Ren, Kathy SM Leung, Eric HY Lau, Jessica Y Wong, et al. Early transmission dynamics in wuhan, china, of novel coronavirus-infected pneumonia. New England Journal of Medicine, 2020a.

Ruiyun Li, Sen Pei, Bin Chen, Yimeng Song, Tao Zhang, Wan Yang, and Jeffrey Shaman. Substantial undocumented infection facilitates the rapid dissemination of novel coronavirus (sars-cov-2). Science, 368(6490):489-493, 2020b.

Alex Lipton and Marcos Lopez de Prado. Exit strategies for covid-19: An application of the k-seir model (presentation slides). Available at SSRN 3579712, 2020.

Maia Martcheva. An introduction to mathematical epidemiology, volume 61. Springer, 2015.

William Hardy McNeill and William McNeill. Plagues and peoples. Anchor, 1998.

Casey B Mulligan. Economic activity and the value of medical innovation during a pandemic. Technical report, National Bureau of Economic Research, 2020.

Cameron Nowzari, Victor M Preciado, and George J Pappas. Optimal resource allocation for control of networked epidemic models. IEEE Transactions on Control of Network Systems, 4(2):159-169, 2015.

NYC Health. Covid-19: Data. https://www1.nyc.gov/site/doh/covid/covid-19-data. page, 2020.

Masaki Ogura and Victor M Preciado. Epidemic processes over adaptive state-dependent networks. Physical Review E, 93(6):062316, 2016.

Masaki Ogura and Victor M Preciado. Optimal containment of epidemics in temporal and adaptive networks. In Temporal Network Epidemiology, pages 241-266. Springer, 2017.

Masaki Ogura, Victor M Preciado, and Naoki Masuda. Optimal containment of epidemics over temporal activity-driven networks. SIAM Journal on Applied Mathematics, 79(3): 986-1006, 2019. 
Pew Research. More than nine-in-ten people worldwide live in countries with travel restrictions amid COVID-19, 2020.

WM Post, DL DeAngelis, and CC Travis. Endemic disease in environments with spatially heterogeneous host populations. Mathematical Biosciences, 63(2):289-302, 1983.

SafeGraph. Covid-19 response datasets, social distancing metrics. https://docs. safegraph.com/docs/social-distancing-metrics, 2020.

The New York Times. Coronavirus (covid-19) data in the united states. https://github. com/nytimes/covid-19-data, 2020.

U.S. Bureau of Labor Statistics. Employment situation summary - april 2020. https: //www.bls.gov/news.release/empsit.nr0.htm, 2020.

US Census Bureau. Tiger/line with selected demographic and economic data. https: //wWw . census.gov/geographies/mapping-files/2010/geo/tiger-data.html, 2010.

US Census Bureau. Longitudinal employer-household dynamics (lodes). https://lehd. ces.census.gov/data/, 2017.

US Census Bureau. Zip code tabulation areas. https : / / www . census . gov / programs-surveys/geography/guidance/geo-areas/zctas.html, 2020.

Pauline Van den Driessche and James Watmough. Reproduction numbers and sub-threshold endemic equilibria for compartmental models of disease transmission. Mathematical biosciences, 180(1-2):29-48, 2002.

Roman Wölfel, Victor M Corman, Wolfgang Guggemos, Michael Seilmaier, Sabine Zange, Marcel A Müller, Daniela Niemeyer, Terry C Jones, Patrick Vollmar, Camilla Rothe, et al. Virological assessment of hospitalized patients with covid-2019. Nature, pages 1-10, 2020.

Joseph T Wu, Kathy Leung, and Gabriel M Leung. Nowcasting and forecasting the potential domestic and international spread of the 2019-ncov outbreak originating in wuhan, china: a modelling study. The Lancet, 395(10225):689-697, 2020. 